\title{
Biangular Lines Revisited
}

\author{
Mikhail Ganzhinov $^{1}$ - Ferenc Szöllősi ${ }^{1}$
}

Received: 15 October 2019 / Revised: 15 September 2020 / Accepted: 11 December 2020 /

Published online: 5 April 2021

(c) The Author(s) 2021

\begin{abstract}
Line systems passing through the origin of the $d$-dimensional Euclidean space admitting exactly two distinct angles are called biangular. It is shown that the maximum cardinality of biangular lines is at least $2(d-1)(d-2)$, and this result is sharp for $d \in\{4,5,6\}$. Connections to binary codes, few-distance sets, and association schemes are explored, along with their multiangular generalization.
\end{abstract}

Keywords Biangular lines $\cdot$ Few-distance sets $\cdot t$-Designs

\section{Introduction}

This paper is concerned with optimal arrangements of unit vectors in Euclidean space. Let $d, m, s \geq 1$ be integers, let $\mathbb{R}^{d}$ denote the $d$-dimensional Euclidean space with standard inner product $\langle\cdot, \cdot\rangle$, and let $\mathscr{X} \subset \mathbb{R}^{d}$ be a set of unit vectors with the associated set of inner products $A(\mathscr{X}):=\left\{\left\langle x, x^{\prime}\right\rangle: x \neq x^{\prime}, x, x^{\prime} \in \mathscr{X}\right\}$. The following two concepts are central to this paper: $\mathscr{X}$ forms a spherical $s$-distance set [5, $34,37,40]$ if $|A(\mathscr{X})| \leq s$; and $\mathscr{X}$ spans a system of $m$-angular lines (passing through the origin in the direction of $x \in \mathscr{X}$ ), if $-1 \notin A(\mathscr{X})$ and $\left|\left\{\gamma^{2}: \gamma \in A(\mathscr{X})\right\}\right| \leq m$. With this terminology a system of $m$-angular lines can be considered as the switching class of certain spherical $2 m$-distance set without antipodal vectors. If the parameters $s$ and $m$ are not specified, then we talk about few-distance sets [9], and multiangular lines, respectively. The fundamental question of interest concerns the maximum cardinality

Editor in Charge: János Pach

This research was supported in part by the Academy of Finland, Grant \#289002.

Mikhail Ganzhinov

mikhail.ganzhinov@aalto.fi

Ferenc Szöllősi

szoferi@gmail.com

1 Department of Communications and Networking, Aalto University School of Electrical Engineering, P.O. Box 15400, 00076 Aalto, Finland 
Table 1 Lower bounds on the maximum number of biangular lines in $\mathbb{R}^{d}$

\begin{tabular}{|c|c|c|c|c|c|c|c|c|c|c|}
\hline$d$ & 2 & 3 & 4 & 5 & 6 & 7 & 8 & 9 & 10 & 11 \\
\hline$\#$ & $5^{*}$ & $10^{*}$ & $12^{*}$ & $24^{*}$ & $40^{*}$ & 72 & 126 & 240 & 256 & 276 \\
\hline$d$ & 12 & 13 & 14 & 15 & 16 & $17-20$ & 21 & 22 & $23-35$ & $36-$ \\
\hline$\#$ & 296 & 336 & 392 & 456 & 576 & 816 & 896 & 1408 & 2300 & $2(d-1)(d-2)$ \\
\hline
\end{tabular}

and structure of the largest sets $\mathscr{X}$ and their corresponding $A(\mathscr{X})$. In particular, one is interested in finding the correct asymptotic growth rate.

Equiangular lines (i.e., the case $m=1$ ) are well-known combinatorial objects $[19,32,33]$, receiving considerable recent attention, see e.g., [3,24]. Biangular lines correspond to the case $m=2$, which have also been the subject of both classical $[15,43]$, as well as more recent studies $[7,8,14,28,41]$. In addition, they have been investigated from the viewpoint of tight frames [23,45]. Our motivation for studying these objects is fueled by their intrinsic connection to kissing arrangements $[16,18,36]$. In particular, we hope that the techniques and results described in this paper will eventually contribute to a deeper understanding of low-dimensional sphere packings. The goal of this paper, which heavily builds on the theory set forth earlier in [44], is to describe a systematic approach to the study of multiangular lines, focusing in particular on the biangular case.

The outline of this paper is as follows: in Sect. 2 we give various constructions of biangular lines, showing that their maximum number is at least $2(d-1)(d-2)$ in $\mathbb{R}^{d}$ for every $d \geq 3$. In Sect. 3 we set up a general computational framework for exhaustively generating all (sufficiently large) biangular line systems, and in Sect. 4 we leverage on these ideas to classify the largest sets in $\mathbb{R}^{d}$ for every $d \leq 6$. In Sect. 5 we present our results on multiangular lines. In Sect. 6 we conclude our manuscript with a selection of open problems. To improve the readability, a technical part on graph representation was moved to Appendix A, along with a few rather large matrices displayed in Appendix B.

For a convenient reference, we display here in Table 1 the best known lower bounds on the maximum number of biangular lines in $\mathbb{R}^{d}$ (where entries marked by $*$ are exact). All these numbers are new, except for the well-known cases in dimensions 2, 3, 22 , and 23.

\section{Constructions of Biangular Lines}

The goal of this section is to give various explicit constructions of large biangular line systems in low dimensional spaces.

Let $\mathscr{X} \subset \mathbb{R}^{d}$ be a set of unit vectors, spanning biangular lines, and let $O$ be an orthogonal matrix representing an isometry of $\mathbb{R}^{d}$. Since for every $x \in \mathscr{X}$ the sets $\mathscr{X}^{\prime}:=(\mathscr{X} \backslash\{x\}) \cup\{-x\}$ and $\mathscr{X}^{\prime \prime}:=\{O x: x \in \mathscr{X}\}$ span the same system of biangular lines as $\mathscr{X}$, we may replace any $x \in \mathscr{X}$ with its negative or apply $O$ 
whenever it is necessary. Throughout this section we represent biangular line systems with a (conveniently chosen) corresponding set of unit vectors, and uniqueness is understood up to these operations. First, we give an elementary proof to the following trivial warm-up result.

Lemma 2.1 The five lines passing through the antipodal vertices of the convex regular decagon form the unique maximum biangular line system in $\mathbb{R}^{2}$.

Proof Let $n \geq 1$, let $\alpha, \beta \in \mathbb{R}$ be such that $0 \leq \alpha<\beta<1$, and assume that $\mathscr{X}:=\left\{x_{i}: i \in\{1, \ldots, n\}\right\}$ spans a maximum biangular line system in $\mathbb{R}^{2}$ with corresponding set of inner products $A(\mathscr{X}) \subseteq\{ \pm \alpha, \pm \beta\}$. We may assume without loss of generality that $x_{1}=[1,0]$. Since for $i \in\{2, \ldots, n\}$ we have $\left\langle x_{1}, x_{i}\right\rangle \in A(\mathscr{X})$, it immediately follows that

$$
x_{i} \in\left\{\left[\alpha, \sqrt{1-\alpha^{2}}\right],\left[\alpha,-\sqrt{1-\alpha^{2}}\right],\left[\beta, \sqrt{1-\beta^{2}}\right],\left[\beta,-\sqrt{1-\beta^{2}}\right]\right\}
$$

after replacing $x_{i}$ by $-x_{i}$ if it is necessary. Therefore $n \leq 5$, and the claimed configuration is indeed a largest possible example.

To see uniqueness, let us use the notation $x_{2}=\left[\alpha, \sqrt{1-\alpha^{2}}\right], x_{3}=\left[\alpha,-\sqrt{1-\alpha^{2}}\right]$, $x_{4}=\left[\beta, \sqrt{1-\beta^{2}}\right]$, and $x_{5}=\left[\beta,-\sqrt{1-\beta^{2}}\right]$. Since $\left\langle x_{2}, x_{3}\right\rangle=2 \alpha^{2}-1,\left\langle x_{4}, x_{5}\right\rangle=$ $2 \beta^{2}-1$, and $\left\langle x_{2}, x_{4}\right\rangle+\left\langle x_{2}, x_{5}\right\rangle=2 \alpha \beta$, the following system of polynomial equations in the variables $\alpha$ and $\beta$ must hold:

$$
\left\{\begin{array}{l}
\left(\left(2 \alpha^{2}-1\right)^{2}-\alpha^{2}\right)\left(\left(2 \alpha^{2}-1\right)^{2}-\beta^{2}\right)=0, \\
\left(\left(2 \beta^{2}-1\right)^{2}-\alpha^{2}\right)\left(\left(2 \beta^{2}-1\right)^{2}-\beta^{2}\right)=0, \\
\alpha \beta\left((\alpha \beta)^{2}-\alpha^{2}\right)\left((\alpha \beta)^{2}-\beta^{2}\right)\left((2 \alpha \beta)^{2}-(\alpha+\beta)^{2}\right)\left((2 \alpha \beta)^{2}-(\alpha-\beta)^{2}\right)=0 .
\end{array}\right.
$$

This admits the unique feasible solution $\alpha=(-1+\sqrt{5}) / 4$ and $\beta=(1+\sqrt{5}) / 4$.

An alternate proof can be given to Lemma 2.1 by using more sophisticated tools, such as Theorem 3.1 and [19, Remark 6.2]. We will show yet another proof later in Sect. 4.

Recall that a binary code of length $d$ with minimum distance $\Delta$ is a set $\mathscr{B} \subseteq \mathbb{F}_{2}^{d}$ such that $\operatorname{dist}\left(b, b^{\prime}\right) \geq \Delta$ for every distinct $b, b^{\prime} \in \mathscr{B}$ where $\operatorname{dist}(\cdot, \cdot)$ denotes the Hamming distance [21]. Let us define the following function:

$$
\Sigma: \mathbb{F}_{2} \rightarrow \mathbb{R}, \quad \Sigma(0)=\frac{1}{\sqrt{d}}, \quad \Sigma(1)=-\frac{1}{\sqrt{d}},
$$

and extend it coordinate-wise to a function from $\mathbb{F}_{2}^{d}$ by writing $\Sigma(b):=\left[\Sigma\left(b_{1}\right), \ldots\right.$, $\left.\Sigma\left(b_{d}\right)\right] \in \mathbb{R}^{d}$. This yields a spherical embedding of the codewords.

Lemma 2.2 Let $d \geq 2$, and let $\Delta_{1}, \Delta_{2} \in\{1, \ldots, d-1\}$. Let $\mathscr{B}$ be a binary code of length $d$, such that $\operatorname{dist}\left(b, b^{\prime}\right) \in\left\{\Delta_{1}, \Delta_{2}, d-\Delta_{1}, d-\Delta_{2}\right\}$ for every distinct $b, b^{\prime} \in \mathscr{B}$. Then $\mathscr{X}:=\{\Sigma(b): b \in \mathscr{B}\}$ spans a system of biangular lines with $A(\mathscr{X}) \subseteq\left\{ \pm\left(1-2 \Delta_{1} / d\right), \pm\left(1-2 \Delta_{2} / d\right)\right\}$.

Proof For every $b, b^{\prime} \in \mathscr{B}$ we have $\left\langle\Sigma(b), \Sigma\left(b^{\prime}\right)\right\rangle=1-2 \operatorname{dist}\left(b, b^{\prime}\right) / d>-1$. 
For terminology and basic facts on lattices we refer the reader to the textbook [18]. It is well known (see [15], [18, p. 117]) that the shortest vectors of the $D_{d}$ lattices give rise to biangular line systems.

Lemma 2.3 Let $d \geq 2$, and let $\mathscr{X} \subset \mathbb{R}^{d}$ be the subset of all permutations of the unit vectors $[ \pm 1, \pm 1,0, \ldots, 0] / \sqrt{2}$ whose first nonzero coordinate is positive. Then $\mathscr{X}$ spans $|\mathscr{X}|=d(d-1)$ biangular lines with $A(\mathscr{X}) \subseteq\{0, \pm 1 / 2\}$.

Proof For distinct $x, x^{\prime} \in \mathscr{X}$ the inner product $\left\langle x, x^{\prime}\right\rangle$ depends on the number of positions where the nonzero coordinates of $x$ and $x^{\prime}$ overlap. If there is no overlap, or there are exactly two overlaps, then $\left\langle x, x^{\prime}\right\rangle=0$. Otherwise, if there is a single overlap, then $\left\langle x, x^{\prime}\right\rangle= \pm 1 / 2$.

Remark 2.4 We remark that for $d \in\{6,7,8\}$ the sets of (nonantipodal) shortest vectors of the exceptional lattices $E_{d}$ give rise to biangular line systems in $\mathbb{R}^{d}$ with inner product set $\{0, \pm 1 / 2\}$ formed by 36,63 , and 120 lines, respectively [18, p. 120].

Let $0<h<1$. Starting from a spherical 2-distance set $\mathscr{X} \subset \mathbb{R}^{d}$, one may obtain a family of biangular line systems in $\mathbb{R}^{d+1}$, where the vectors $x \in \mathscr{X}$ are rescaled by a factor of $\sqrt{1-h^{2}}$ and translated along the $(d+1)$ st coordinate to height $h$. In a similar spirit, the six diagonals of the icosahedron can be continuously twisted in $\mathbb{R}^{3}$, yielding a family of biangular lines [14].

Proposition 2.5 (infinite families) Let $\mathscr{X} \subset \mathbb{R}^{d}$ be a spherical 2-distance set with $A(\mathscr{X}) \subseteq\{\alpha, \beta\}$, with $\alpha, \beta \geq-1$ and $\alpha, \beta<1$. Let $0<h<1$. Then

$$
\mathscr{Y}(h):=\left\{\left[h, x \sqrt{1-h^{2}}\right]: x \in \mathscr{X}\right\}
$$

spans a system of biangular lines in $\mathbb{R}^{d+1}$ with $A(\mathscr{Y}(h)) \subseteq\left\{h^{2}+\left(1-h^{2}\right) \alpha, h^{2}+\right.$ $\left.\left(1-h^{2}\right) \beta\right\}$.

Proof For every $y, y^{\prime} \in \mathscr{Y}(h)$ we have $\left\langle y, y^{\prime}\right\rangle=h^{2}+\left(1-h^{2}\right)\left\langle x, x^{\prime}\right\rangle$ for some $x, x^{\prime} \in \mathscr{X}$. Furthermore, $-1 \notin A(\mathscr{Y}(h))$ by our assumptions on $h$.

Since the midpoints of the edges of the regular simplex in $\mathbb{R}^{d}$ form a spherical 2distance set of size $d(d+1) / 2$, biangular lines of this cardinality are abundant in $\mathbb{R}^{d+1}$. Translation to a well-chosen height yields the following.

Proposition 2.6 (lifting) Let $\mathscr{X} \subset \mathbb{R}^{d}$ be a spherical 4-distance set with $A(\mathscr{X}) \subseteq$ $\{\alpha, \beta, \gamma, \alpha+\beta-\gamma\}$, with $\alpha, \beta, \gamma \geq-1$ and $\alpha, \beta, \gamma<1$, and assume that $\alpha+\beta<0$. Then $\mathscr{Y}:=\{[\sqrt{-\alpha-\beta}, x \sqrt{2}] / \sqrt{2-\alpha-\beta}: x \in \mathscr{X}\}$ spans a system of biangular lines in $\mathbb{R}^{d+1}$ with

$$
A(\mathscr{Y}) \subseteq\left\{ \pm \frac{\alpha-\beta}{2-\alpha-\beta}, \pm \frac{2 \gamma-\alpha-\beta}{2-\alpha-\beta}\right\} .
$$

Proof For every $y, y^{\prime} \in \mathscr{Y}$ we have $\left\langle y, y^{\prime}\right\rangle=\left(-(\alpha+\beta)+2\left\langle x, x^{\prime}\right\rangle\right) /(2-\alpha-\beta)$ for some $x, x^{\prime} \in \mathscr{X}$. Furthermore, $-1 \notin A(\mathscr{Y})$ by our assumptions on $\alpha, \beta, \gamma$. 
Remark 2.7 Given a spherical 3-distance set $\mathscr{X}$ with $A(\mathscr{X}) \subseteq\{\alpha, \beta, \gamma\}$, it might happen that $\alpha+\beta<0, \alpha+\gamma<0$, and $\beta \neq \gamma$. When this occurs, lifting via Proposition 2.6 could result in nonisometric biangular line systems.

The main utility of Proposition 2.6 is that antipodal vectors (which span exactly the same line) can be split into two nonantipodal vectors in the space one dimension higher. It immediately follows that any equiangular line system leads to twice as many biangular lines in the space one dimension higher.

Theorem 2.8 (cf. Theorem 3.1) For every $d \geq 3$, there exists a set $\mathscr{X} \subset \mathbb{R}^{d}$ spanning $|\mathscr{X}|=2(d-1)(d-2)$ biangular lines with $A(\mathscr{X}) \subseteq\{ \pm 1 / 5, \pm 3 / 5\}$.

Proof Take all $2(d-1)(d-2)$ vectors in $\mathbb{R}^{d-1}$ forming a spherical 4-distance set $\mathscr{X}$ with $A(\mathscr{X}) \subseteq\{-1,-1 / 2,0,1 / 2\}$ in Lemma 2.3, and then use Proposition 2.6 to get the claimed biangular line systems.

A further application of Proposition 2.6 is the following.

Corollary 2.9 For $d \in\{4, \ldots, 16\}$ there exists a set $\mathscr{X} \subset \mathbb{R}^{d}$ spanning $|\mathscr{X}|=\left(\begin{array}{c}d \\ 3\end{array}\right)$ biangular lines. There exists a set $\mathscr{X} \subset \mathbb{R}^{17}$ spanning $|\mathscr{X}|=\left(\begin{array}{c}18 \\ 3\end{array}\right)$ biangular lines.

Proof Consider the 'canonical' spherical 3-distance set $\mathscr{X}$ of cardinality $\left(\begin{array}{l}d \\ 3\end{array}\right)$ which can be obtained from

$$
\sqrt{\frac{d-3}{3 d}}\left[1,1,1,-\frac{3}{d-3}, \ldots,-\frac{3}{d-3}\right] \in \mathbb{R}^{d}
$$

by permuting the coordinates. It is easily seen that

$$
A(\mathscr{X}) \subseteq\left\{-\frac{3}{d-3}, \frac{d-9}{3(d-3)}, \frac{2 d-9}{3(d-3)}\right\} .
$$

Since for every $x \in \mathscr{X},\langle x,[1,1, \ldots, 1]\rangle=0, \mathscr{X}$ is embedded into $\mathbb{R}^{d-1}$. Consequently, if $d=18$ then $\mathscr{X}$ spans a biangular line system in $\mathbb{R}^{17}$. If $d \leq 16$, then since $(d-9) /(3(d-3))-3 /(d-3)<0$, Proposition 2.6 yields the claimed configurations in $\mathbb{R}^{d}$.

Finally, a rather surprising consequence of Proposition 2.6 is the following: the biangular line systems mentioned in Remark 2.4 are not the best possible in their respective dimension.

Corollary 2.10 There exists a set $\mathscr{X} \subset \mathbb{R}^{d}$ spanning biangular lines with $A(\mathscr{X}) \subseteq$ $\{ \pm 1 / 5, \pm 3 / 5\}$ for

$$
(d,|\mathscr{X}|) \in\{(3,4),(4,12),(5,24),(6,40),(7,72),(8,126),(9,240)\} .
$$

Proof The cases $d \in\{3,4,5,6\}$ follow from Theorem 2.8. To see the remaining cases, combine Proposition 2.6 with the exceptional configurations mentioned in Remark 2.4. 
Later (see Sect. 4) we will show that Theorem 2.8 gives rise to a largest possible line system for $d \in\{4,5,6\}$, and we tend to believe that Corollary 2.10 gives the best configurations for $d \in\{7,8,9\}$ as well.

Next we prove a preliminary technical result. Following the terminology of [32], we denote by $N_{1 / 3}(d)$ the maximum number of equiangular lines in $\mathbb{R}^{d}$ where the set of inner products is a subset of $\{ \pm 1 / 3\}$. Recall that $N_{1 / 3}(0)=0$.

Proposition 2.11 For $m \geq 1$ and $d \geq m$, there exists a set $\mathscr{X} \subset \mathbb{R}^{d}$ spanning $|\mathscr{X}|=2 m \cdot N_{1 / 3}(d-m)$ biangular lines with $A(\mathscr{X}) \subseteq\{ \pm 1 / 5, \pm 3 / 5\}$.

Proof Let $\mathscr{E}$ denote the set of canonical basis vectors of $\mathbb{R}^{m}$, and consider a maximum set $\mathscr{Y} \subset \mathbb{R}^{d-m}$ spanning $N_{1 / 3}(d-m)$ equiangular lines with $A(\mathscr{Y}) \subseteq\{ \pm 1 / 3\}$. We claim that the following set $\mathscr{X} \subset \mathbb{R}^{d}$ spans a biangular line system:

$$
\mathscr{X}:=\left\{\frac{[y \sqrt{6}, 2 e]}{\sqrt{10}}: y \in \mathscr{Y}, e \in \mathscr{E}\right\} \cup\left\{\frac{[y \sqrt{6},-2 e]}{\sqrt{10}}: y \in \mathscr{Y}, e \in \mathscr{E}\right\} .
$$

Indeed, as for $x, x^{\prime} \in \mathscr{X}$, we have $\left\langle x, x^{\prime}\right\rangle=3\left\langle y, y^{\prime}\right\rangle / 5 \pm 2\left\langle e, e^{\prime}\right\rangle / 5$ for some (not necessarily distinct) $e, e^{\prime} \in \mathscr{E}$ and $y, y^{\prime} \in \mathscr{Y}$. Since $\left\langle e, e^{\prime}\right\rangle \in\{0,1\}$ and $\left\langle y, y^{\prime}\right\rangle \in$ $\{ \pm 1 / 3,1\}$, the claim follows.

We note the following.

Corollary 2.12 There exists a set $\mathscr{X} \subset \mathbb{R}^{14}$ spanning $|\mathscr{X}|=392$ biangular lines with $A(\mathscr{X}) \subseteq\{ \pm 1 / 5, \pm 3 / 5\}$.

Proof It follows from Proposition 2.11 by setting $m=7$ and $d=7$, and by recalling from [32] that $N_{1 / 3}(7)=28$.

It turns out that one may combine certain line systems described in Proposition 2.11 with the 256 lines spanned by the 'even half' of the 10-dimensional hypercube. This yields improved results for $d \in\{10,11,12,13,15,16\}$ and gives the same number of biangular lines for $d=17$ as Corollary 2.9.

Theorem 2.13 For $d \geq 10$, there exists a set $\mathscr{X} \subset \mathbb{R}^{d}$ spanning $|\mathscr{X}|=256+$ $20 N_{1 / 3}(d-10)$ biangular lines with $A(\mathscr{X}) \subseteq\{ \pm 1 / 5, \pm 3 / 5\}$.

Proof Let $\mathscr{B} \subset \mathbb{F}_{2}^{10}$ be the binary code of length 10 formed by codewords of even weight, such that the first coordinate of every $b \in \mathscr{B}$ is 0 . By Lemma 2.2 the set $\mathscr{Z}:=\{\Sigma(b): b \in \mathscr{B}\} \subset \mathbb{R}^{10}$ spans a system of 256 biangular lines with $A(\mathscr{Z}) \subseteq$ $\{ \pm 1 / 5, \pm 3 / 5\}$. Next, we consider a maximum set $\mathscr{Y} \subset \mathbb{R}^{d-10}$ spanning $N_{1 / 3}(d-10)$ equiangular lines with $A(\mathscr{Y}) \subseteq\{ \pm 1 / 3\}$. Let $\mathscr{E}$ denote the set of canonical basis vectors of $\mathbb{R}^{10}$, and let $o \in \mathbb{R}^{d-10}$ denote the zero vector. We claim that the following set $\mathscr{X} \subset \mathbb{R}^{d}$ spans a biangular line system:

$$
\begin{aligned}
\mathscr{X}:= & \{[\sqrt{6} y, 2 e] / \sqrt{10}: y \in \mathscr{Y}, e \in \mathscr{E}\} \\
& \cup\{[\sqrt{6} y,-2 e] / \sqrt{10}: y \in \mathscr{Y}, e \in \mathscr{E}\} \cup\{[o, z]: z \in \mathscr{Z}\} .
\end{aligned}
$$


Indeed, for $x, x^{\prime} \in \mathscr{X}$, we have

$$
\left\langle x, x^{\prime}\right\rangle \in\left\{3\left\langle y, y^{\prime}\right\rangle / 5 \pm 2\left\langle e, e^{\prime}\right\rangle / 5, \pm 2\langle e, z\rangle / \sqrt{10},\left\langle z, z^{\prime}\right\rangle\right\}
$$

for some (not necessarily distinct) $e, e^{\prime} \in \mathscr{E}, y, y^{\prime} \in \mathscr{Y}$, and $z, z^{\prime} \in \mathscr{Z}$. Since $\left\langle e, e^{\prime}\right\rangle \in$ $\{0,1\},\langle e, z\rangle \in\{ \pm 1 / \sqrt{10}\},\left\langle y, y^{\prime}\right\rangle \in\{ \pm 1 / 3,1\}$, and $\left\langle z, z^{\prime}\right\rangle \in\{ \pm 1 / 5, \pm 3 / 5,1\}$, the claim follows.

Corollary 2.14 There exists a set $\mathscr{X} \subset \mathbb{R}^{d}$ spanning biangular lines with $A(\mathscr{X}) \subseteq$ $\{ \pm 1 / 5, \pm 3 / 5\}$ for

$$
\begin{array}{r}
(d,|\mathscr{X}|) \in\{(10,256),(11,276),(12,296),(13,336), \\
(15,456),(16,576),(17,816)\} .
\end{array}
$$

Proof Combine Theorem 2.13 with [32, Thm. 4.5].

Finally, we note that various cross-sections of the Leech lattice $\Lambda_{24}$ (see [18, p. 133] for how to construct its shortest vectors from the extended binary Golay code [12] in explicit form) give rise to biangular line systems with inner product set $\{0, \pm 1 / 3\}$. Such line systems were investigated in [43].

Theorem 2.15 There exists a set $\mathscr{X} \subset \mathbb{R}^{d}$ spanning biangular lines with $A(\mathscr{X}) \subseteq$ $\{0, \pm 1 / 3\}$ for $(d,|\mathscr{X}|) \in\{(21,896),(22,1408),(23,2300)\}$.

Proof Let $\mathscr{L} \subset \mathbb{R}^{24},|\mathscr{L}|=196560$, be the set of shortest vectors of $\Lambda_{24}$, where the vectors are normalized so that $\langle\ell, \ell\rangle=1$ for every $\ell \in \mathscr{L}$. With this convention, $\left\langle\ell, \ell^{\prime}\right\rangle \in\{0, \pm 1 / 4, \pm 1 / 2, \pm 1\}$ for every $\ell, \ell^{\prime} \in \mathscr{L}$. Now let $\ell \in \mathscr{L}$ be fixed. It is well known (see [18, p. 264]) that the subset $\mathscr{Y}=\{y:\langle\ell, y\rangle=1 / 2, y \in \mathscr{L}\}$ contains 4600 vectors, independently of the choice of $\ell$. Note that for $y \in \mathscr{Y}$ we have $\ell-y \in \mathscr{Y}$ and therefore the set $\mathscr{Z}:=\{(2 y-\ell) / \sqrt{3}: y \in \mathscr{Y}\}$ is antipodal, and $\langle\ell, z\rangle=0$ for every $z \in \mathscr{Z}$. Finally, let $\mathscr{X} \subset \mathscr{Z}$ with $|\mathscr{X}|=2300$ so that $\mathscr{Z}=\{x: x \in \mathscr{X}\} \cup\{-x: x \in \mathscr{X}\}$. Now $\mathscr{X}$ spans the claimed biangular line system in $\mathbb{R}^{23}$, since $\left\langle y, y^{\prime}\right\rangle \notin\{-1 / 4,-1\}$ and therefore for $x, x^{\prime} \in \mathscr{X}$ we have $\left\langle x, x^{\prime}\right\rangle=\left(4\left\langle y, y^{\prime}\right\rangle-1\right) / 3 \in\{0, \pm 1 / 3,1\}$. Let $x, x^{\prime} \in \mathscr{X}$ be such that $\left\langle x, x^{\prime}\right\rangle=0$. Then $\mathscr{U}:=\{u:\langle u, x\rangle=0, u \in \mathscr{X}\}, \mathscr{V}:=\left\{v:\langle v, x\rangle=\left\langle v, x^{\prime}\right\rangle=0, v \in \mathscr{X}\right\}$ span the claimed biangular line systems in dimension 22 and 21 , respectively.

Another way to get biangular lines with the set of inner products $\{0, \pm 1 / 3\}$ is the following.

Lemma 2.16 Let $w \equiv 3(\bmod 4)$ and $d \geq 2 w+1$ be positive integers. Let $\mathscr{B} \subset \mathbb{F}_{2}^{d}$ be a binary constant weight code of length $d$, weight $w$, and minimum distance $2 w-2$, and assume that there exists a Hadamard matrix of order $w+1$. Then there exists a set $\mathscr{X} \subset \mathbb{R}^{d}$ with $|\mathscr{X}|=(w+1)|\mathscr{B}|$ spanning a biangular line system with $A(\mathscr{X}) \subseteq\{0, \pm 1 / w\}$.

Proof Recall that a Hadamard matrix $H$ of order $w+1$ is a $(w+1) \times(w+1)$ orthogonal matrix with entries $\pm 1 / \sqrt{w+1}$. Let $H^{\prime}$ be the matrix obtained from $H$ 
after removing its first column and renormalizing its rows. Let $\mathscr{H} \subset \mathbb{R}^{w}$ be the set of rows of $H^{\prime}$. Clearly, $\left\langle h, h^{\prime}\right\rangle \in\{ \pm 1 / w, 1\}$ for $h, h^{\prime} \in \mathscr{H}$. Now $\mathscr{X}$ can be obtained by replacing each codeword $b \in \mathscr{B}$ with a set of $w+1$ real vectors where the support of $b$ (i.e., coordinates with binary 1) are replaced by the entries of $h \in \mathscr{H}$, and coordinates with binary 0 are replaced by $0 \in \mathbb{R}$. Since $d \geq 2 w+1$, there are no two codewords at Hamming distance $d$, and therefore the claim follows.

Corollary 2.17 For $d \geq 7$ there exists a set $\mathscr{X} \subset \mathbb{R}^{d}$ spanning $|\mathscr{X}|=4\lceil(d-1)$ $(d-2) / 6\rceil$ biangular lines with $A(\mathscr{X}) \subseteq\{0, \pm 1 / 3\}$. Furthermore, there exists a set $\mathscr{Y} \subset \mathbb{R}^{d+1}$ spanning $|\mathscr{X}|$ biangular lines with $A(\mathscr{Y}) \subseteq\{ \pm 1 / 7, \pm 3 / 7\}$.

Proof Indeed, this is a specialization of Lemma 2.16 for $w=3$ and using constant weight codes coming from the averaging argument in [13, Thm. 14]. The second part of the claim is an immediate consequence of Proposition 2.6.

While Corollary 2.17 is weaker than Theorem 2.8, it can be used in two ways. First, one may embed the 2300 biangular lines from Theorem 2.15 into $\mathbb{R}^{23+d}$, and extend this configuration with an additional $4\lceil(d-1)(d-2) / 6\rceil$ vectors (for $d \geq 7$ ). Secondly, it may happen that these configurations can be further extended to a spherical 4-distance set with inner products $\{-2 / 3,-1 / 3,0,1 / 3\}$, and then an application of Proposition 2.6 would immediately yield biangular lines with inner products $\{ \pm 1 / 7, \pm 3 / 7\}$ in $\mathbb{R}^{24+d}$. The following result shows that the two largest sets mentioned in Theorem 2.15 are inextendible.

Theorem 2.18 (the relative bound, [10,19]) Let $d \geq 3$ and assume that $\mathscr{X} \subset \mathbb{R}^{d}$ spans a biangular line system with $A(\mathscr{X}) \subseteq\{ \pm \alpha, \pm \beta\}, 0 \leq \alpha, \beta<1$. Assume that $\alpha^{2}+\beta^{2} \leq 6 /(d+4)$ and $3-(d+2)\left(\alpha^{2}+\beta^{2}\right)+d(\bar{d}+2) \alpha^{2} \beta^{2}>0$. Let $n_{\alpha}:=\left|\left\{\left[x, x^{\prime}\right]:\left\langle x, x^{\prime}\right\rangle^{2}=\alpha^{2}, x, x^{\prime} \in \mathscr{X}\right\}\right|$. Then

$$
|\mathscr{X}| \leq \frac{d(d+2)\left(1-\alpha^{2}\right)\left(1-\beta^{2}\right)}{3-(d+2)\left(\alpha^{2}+\beta^{2}\right)+d(d+2) \alpha^{2} \beta^{2}} .
$$

Equality holds if and only if

$$
\begin{aligned}
&\left(\frac{6}{d+4}-\alpha^{2}-\beta^{2}\right)\left(\left(\alpha^{2}-\beta^{2}\right) n_{\alpha}+|\mathscr{X}|(|\mathscr{X}|-1) \beta^{2}+|\mathscr{X}|-\frac{|\mathscr{X}|^{2}}{d}\right)=0 \text { and } \\
&\left(\frac{6}{d+4}-\alpha^{2}-\beta^{2}\right)\left(\alpha^{2}-\beta^{2}\right) n_{\alpha} \\
&=\frac{|\mathscr{X}|\left(d^{2}+3|\mathscr{X}|-4\right)}{(d+2)(d+4)}-|\mathscr{X}|(|\mathscr{X}|-1) \beta^{2}\left(\frac{6}{d+4}-\beta^{2}\right) .
\end{aligned}
$$

Remark 2.19 For $d \geq 3$ and $i \in\{2,4\}$ let $C_{i}^{((d-2) / 2)}(z)$ denote the Gegenbauer polynomials in the sense of [20]. In Theorem 2.18 the equality holds if and only if

$$
\left(\frac{6}{d+4}-\alpha^{2}-\beta^{2}\right) \sum_{x, x^{\prime} \in \mathscr{X}} C_{2}^{((d-2) / 2)}\left(\left\langle x, x^{\prime}\right\rangle\right)=\sum_{x, x^{\prime} \in \mathscr{X}} C_{4}^{((d-2) / 2)}\left(\left\langle x, x^{\prime}\right\rangle\right)=0
$$


In particular, if $\mathscr{X} \subset \mathbb{R}^{d}$ forms a spherical 4-design [4] such that $A(\mathscr{X}) \subseteq\{ \pm \alpha, \pm \beta\}$, then the equality holds in (1).

Remark 2.20 If there is equality in (1), then the quantity $n_{\alpha}$ as defined in Theorem 2.18 is a nonnegative integer. The failure of this condition could be used to show the nonexistence of various hypothetical configurations, e.g., in $\mathbb{R}^{8}$ there does not exist 50 biangular lines with the set of inner products $\{ \pm 1 / 4, \pm 1 / 2\}$.

In Table 2 we display data on the known biangular line systems meeting the relative bound, and later in Corollary 4.12 we prove that this list is (essentially) complete for $d \leq 6$. The canonical examples are mutually unbiased bases (MUBs) [29], spanning $2^{4 i-1}+2^{2 i}$ biangular lines in dimension $d=4^{i}$ with inner products $\left\{0, \pm 2^{-i}\right\}, i \geq 1$. We note the following two examples.

Example 2.21 (36 biangular lines in $\mathbb{R}^{7}$, see [17,22]) Let $U$ be the $7 \times 7$ circulant matrix with first row $[0,1,0,0,0,0,0]$. Let

$$
\begin{aligned}
\mathscr{Y} & :=\{[-7,1,1,1,1,1,1],[-1,3,3,-3,3,-3,-3]\} \text { and } \\
\mathscr{Z}:= & \{[1,-1,-3,3,3,-3,-3],[1,3,-1,-3,-3,-3,3], \\
& \quad[-1,3,-3,1,-3,3,-3]\} .
\end{aligned}
$$

Then, the set

$$
\begin{array}{r}
\mathscr{X}=\{[-7,1,1,1,1,1,1,1] / \sqrt{56}\} \cup\left\{\left[1, y U^{i}\right] / \sqrt{56}: i \in\{0,1, \ldots, 6\}, y \in \mathscr{Y}\right\} \\
\cup\left\{\left[3, z U^{i}\right] / \sqrt{56}: i \in\{0,1, \ldots, 6\}, z \in \mathscr{Z}\right\}
\end{array}
$$

spans 36 biangular lines in $\mathbb{R}^{7}$ with $A(\mathscr{X}) \subseteq\{ \pm 1 / 7, \pm 3 / 7\}$. Indeed, all vectors are orthogonal to $[1, \ldots, 1] \in \mathbb{R}^{8}$. The parameters of this line system meet the relative bound.

Example 2.22 (256 biangular lines in $\mathbb{R}^{16}$, see [21, p. 486], [38], [43]) Consider a biplane [31] of order 4 , that is, a $16 \times 16$ square $\{0,1\}$-matrix $H$ with constant row and column sum 6 , such that $H H^{T}=4 I_{16}+2 J_{16}$. We may simply take $H:=$ $\left(J_{4}-I_{4}\right) \otimes I_{4}+I_{4} \otimes\left(J_{4}-I_{4}\right)$, and let $\mathscr{H} \subset \mathbb{R}^{16}$ be the set of rows of $H$. Let $\mathscr{B} \subset \mathbb{F}_{2}^{6}$ be a binary code of length 6 formed by codewords of even weight, such that the first coordinate of every $b \in \mathscr{B}$ is 0 . By Lemma 2.2 the set $\mathscr{Z}:=\{\Sigma(b): b \in \mathscr{B}\} \subset \mathbb{R}^{6}$ spans a system of 16 biangular lines with $A(\mathscr{Z}) \subseteq\{ \pm 1 / 3\}$. Replacing each codeword $b \in \mathscr{B}$ with a set of 16 real vectors where the support of $b$ (i.e., coordinates with binary 1) are replaced by the entries of $h \in \mathscr{H}$, and coordinates with binary 0 are replaced by $0 \in \mathbb{R}$, spans the claimed 256 biangular lines in $\mathbb{R}^{16}$ with the set of inner products $\{0, \pm 1 / 3\}$. Further nonisometric examples can be constructed by, e.g., choosing $H$ in a different way. The parameters of these line systems meet the relative bound. ${ }^{1}$

\footnotetext{
1 We are indebted to the reviewers for pointing out references. Apparently, Example 2.22 is related to the minimal vectors of an overlattice of the Barnes-Wall lattice $B W_{16}$, as described on the webpage http:// www.math.rwth-aachen.de/ Gabriele.Nebe/LATTICES/obw16.html.
} 
Table 2 Biangular line systems meeting the relative bound

\begin{tabular}{llll}
\hline$d$ & $n$ & $\{\alpha, \beta\}$ & Remark \\
\hline 3 & 6 & $\{ \pm 1 / \sqrt{5}\}$ & Icosahedron \\
& 10 & $\{ \pm 1 / 3, \pm \sqrt{5} / 3\}$ & Dodecahedron \\
4 & 12 & $\{0, \pm 1 / 2\}$ & $D_{4}$ lattice (MUBs) \\
6 & 27 & $\{ \pm 1 / 4, \pm 1 / 2\}$ & Schläfli graph, Example B.3 \\
& 36 & $\{0, \pm 1 / 2\}$ & $E_{6}$ lattice \\
7 & 28 & $\{ \pm 1 / 3\}$ & Equiangular lines \\
& 36 & $\{ \pm 1 / 7, \pm 3 / 7\}$ & Example 2.21 \\
& 63 & $\{0, \pm 1 / 2\}$ & $E_{7}$ lattice \\
16 & 120 & $\{0, \pm 1 / 2\}$ & $E_{8}$ lattice \\
& 144 & $\{0, \pm 1 / 4\}$ & MUBs \\
22 & 256 & $\{0, \pm 1 / 3\}$ & Example 2.22 \\
& 275 & $\{ \pm 1 / 6, \pm 1 / 4\}$ & McLaughlin graph \\
23 & 1408 & $\{0, \pm 1 / 3\}$ & From $\Lambda_{24}$ \\
& 276 & $\{ \pm 1 / 5\}$ & Equiangular lines \\
$4^{i}$ & 2300 & $\{0, \pm 1 / 3\}$ & From $\Lambda_{24}$ \\
\hline & $24-1+2^{2 i}$ & $\{0, \pm 2-i\}$ & MUBs, $i \geq 3$ \\
\hline
\end{tabular}

Finally, we note the following (almost immediate) consequence of [39, Theorem 5.2 and 5.3].

Theorem 2.23 (see [39]) Let $d \geq 5$ and let $\mathscr{X} \subset \mathbb{R}^{d}$ span a maximum biangular line system with $A(\mathscr{X}) \subseteq\{ \pm \alpha, \pm \beta\}, 0 \leq \alpha<\beta<1$. Then $z:=\left(1-\alpha^{2}\right) /\left(\beta^{2}-\alpha^{2}\right)$ is an integer. Furthermore,

$$
z \leq\left\lfloor\frac{1}{2}+\sqrt{\frac{\left(d^{2}+d+2\right)\left(d^{2}+d-1\right)}{4 d^{2}+4 d-8}}\right\rfloor .
$$

Proof The statement is a reformulation of [39, Thms. 5.2 and 5.3] and it holds whenever $|\mathscr{X}| \geq d(d+1)$. This in turn holds by Theorem 2.8 for maximum biangular line systems whenever $d \geq 7$. For $d \in\{5,6\}$ the set of inner products of (the unique) maximum biangular line systems is $\{ \pm 1 / 5, \pm 3 / 5\}$ (see Theorems 4.7 and 4.9), and therefore in these cases $z=3$ is indeed an integer below the claimed bound.

\section{Computational Framework}

In this section, following ideas developed in [44], we set up a framework for systematically generating biangular lines. We will leverage on this newly established theory in Sect. 4 where we demonstrate how to use this approach in practice. In particular, we will determine the size of the largest biangular line systems in dimension $d \leq 6$ by using supercomputational resources, and classify the maximum cases. We remark that this framework carries over to the multiangular setting after minor technical changes (see Sect. 5 and Appendix A). 


\subsection{A High Level Overview}

Let $d, n \geq 1$, and let $\mathscr{X}=\left\{x_{1}, \ldots, x_{n}\right\} \subset \mathbb{R}^{d}$ be a set of unit vectors, spanning a system of $n$ biangular lines. From here on, we will represent $\mathscr{X}$ by its Gram matrix $G:=\left[\left\langle x_{i}, x_{j}\right\rangle\right]_{i, j=1}^{n}$. Conveniently, the matrix $G$ is invariant up to change of basis, and has the following combinatorial properties: $G$ is $n \times n, G=G^{T}, G_{i i}=1$ for every $i \in\{1, \ldots, n\}$, and $G_{i j} \in A(\mathscr{X})$ for distinct $i, j \in\{1, \ldots, n\}$. Furthermore, it has the following algebraic properties: $G$ is positive-semidefinite and rank $G \leq d$. Conversely, given any matrix $G$ with these properties, one may reconstruct (uniquely, up to change of basis), via the Cholesky decomposition, an $n \times \operatorname{rank} G$ matrix $F$ such that $F F^{T}=G$ holds [27].

Our aim is to find a way for generating all (sufficiently large) $n \times n$ Gram matrices of biangular line systems in a fixed dimension $d$. It follows from Ramsey theory that $n$ is bounded in terms of $d$, and we recall here the following explicit upper bound.

Theorem 3.1 (absolute bound, [19], cf. Theorem 2.8) Let $\mathscr{X} \subset \mathbb{R}^{d}$ span a biangular line system. Then $|\mathscr{X}| \leq\left(\begin{array}{c}d+3 \\ 4\end{array}\right)$.

We say that a permutation $\sigma$ of the set $\Gamma=\{\alpha, \beta,-\alpha,-\beta\}$ is a relabeling if $\sigma(\gamma)=$ $-\sigma(-\gamma)$ for every $\gamma \in \Gamma$. The following is central to this paper.

Definition 3.2 Let $C(\alpha, \beta)$ be an $n \times n$ symmetric matrix with constant diagonal 1 over the polynomial ring $\mathbb{Q}[\alpha, \beta]$ whose off-diagonal entries are $\{0, \pm \alpha, \pm \beta\}$. Two such matrices, $C_{1}$ and $C_{2}$, are called equivalent, if $C_{1}(\alpha, \beta)=P C_{2}(\sigma(\alpha), \sigma(\beta)) P^{T}$ for some signed permutation matrix $P$ and relabeling $\sigma$. A representative of this matrix equivalence class is called a candidate Gram matrix.

Candidate Gram matrices capture the combinatorial structure of Gram matrices. Since our focus is on the biangular case, we will assume in the following that

$$
\alpha \beta\left(\alpha^{2}-\beta^{2}\right)\left(\alpha^{2}-1\right)\left(\beta^{2}-1\right) \neq 0 .
$$

Furthermore, at most two out of the three symbols $0, \pm \alpha, \pm \beta$ can appear as a matrix entry in $C(\alpha, \beta)$. Clearly, if $G$ is a Gram matrix of a biangular line system, then there exists a candidate Gram matrix $C(\alpha, \beta)$ such that $G=C\left(\alpha^{*}, \beta^{*}\right)$ for some $\alpha^{*}, \beta^{*} \in \mathbb{R}$, subject to (2). In particular, rank $C\left(\alpha^{*}, \beta^{*}\right) \leq d$ should hold.

Example 3.3 (candidate Gram matrices of order 3)

$$
\left[\begin{array}{lll}
1 & 0 & 0 \\
0 & 1 & 0 \\
0 & 0 & 1
\end{array}\right],\left[\begin{array}{lll}
1 & 0 & 0 \\
0 & 1 & \alpha \\
0 & \alpha & 1
\end{array}\right],\left[\begin{array}{lll}
1 & 0 & \alpha \\
0 & 1 & \alpha \\
\alpha & \alpha & 1
\end{array}\right],\left[\begin{array}{ccc}
1 & \alpha & \alpha \\
\alpha & 1 & \alpha \\
\alpha & \alpha & 1
\end{array}\right],\left[\begin{array}{lll}
1 & \alpha & \alpha \\
\alpha & 1 & \beta \\
\alpha & \beta & 1
\end{array}\right]
$$

Note that at most two symbols appear (whose values are unspecified) within the offdiagonal positions, signifying distinct inner products.

The main advantage of using candidate Gram matrices is that in this way we are transforming the problem of 'infinitely many $n \times n$ Gram matrices' to the conceptually 
simpler 'finite list of $n \times n$ candidate Gram matrices' (where $n$ itself is bounded by Theorem 3.1). Then, one should decide whether a candidate Gram matrix actually represents a Gram matrix via a spectral analysis, as illustrated below.

Example 3.4 (Petersen graph, cf. Proposition 2.5) Consider the following example of a candidate Gram matrix of order 10:

$$
C(\alpha, \beta)=\left[\begin{array}{cccccccccc}
1 & \alpha & \alpha & \alpha & \alpha & \alpha & \alpha & \beta & \beta & \beta \\
\alpha & 1 & \alpha & \alpha & \alpha & \beta & \beta & \alpha & \alpha & \beta \\
\alpha & \alpha & 1 & \alpha & \beta & \alpha & \beta & \alpha & \beta & \alpha \\
\alpha & \alpha & \alpha & 1 & \beta & \beta & \alpha & \beta & \alpha & \alpha \\
\alpha & \alpha & \beta & \beta & 1 & \alpha & \alpha & \alpha & \alpha & \beta \\
\alpha & \beta & \alpha & \beta & \alpha & 1 & \alpha & \alpha & \beta & \alpha \\
\alpha & \beta & \beta & \alpha & \alpha & \alpha & 1 & \beta & \alpha & \alpha \\
\beta & \alpha & \alpha & \beta & \alpha & \alpha & \beta & 1 & \alpha & \alpha \\
\beta & \alpha & \beta & \alpha & \alpha & \beta & \alpha & \alpha & 1 & \alpha \\
\beta & \beta & \alpha & \alpha & \beta & \alpha & \alpha & \alpha & \alpha & 1
\end{array}\right]
$$

Here $C(0,1)-I_{10}$ is the adjacency matrix of the Petersen graph. Using standard spectral graph theory, one may find that for every $\alpha^{*}, \beta^{*} \in \mathbb{R}$ we have $\Lambda\left(C\left(\alpha^{*}, \beta^{*}\right)\right)=\left\{\left[1+6 \alpha^{*}+3 \beta^{*}\right]^{1},\left[1+\alpha^{*}-2 \beta^{*}\right]^{4},\left[1-2 \alpha^{*}+\beta^{*}\right]^{5}\right\}$. Therefore rank $C\left(\alpha^{*}, 2 \alpha^{*}-1\right) \leq 5$. Furthermore, for $\alpha^{*} \geq 1 / 6, \alpha^{*}<1$ the matrix $C\left(\alpha^{*}, 2 \alpha^{*}-1\right)$ is positive semidefinite. The matrix $C(1 / 6,-2 / 3)$ on the boundary describes the Petersen code [2], which corresponds to the midpoints of the regular simplex in $\mathbb{R}^{4}$. We remark that since the Petersen code is a 2-distance set, Proposition 2.5 can be applied which gives rise to the family of biangular lines $C\left(\alpha^{*}, 2 \alpha^{*}-1\right)$ in $\mathbb{R}^{5}$.

However, computing the spectrum of a candidate Gram matrix without any apparent structure is a delicate task, and instead we will rely on the following key technical result.

Proposition 3.5 (strong Gröbner test, cf. Corollary 3.8) Let $d \geq 2$ be fixed and let $C(\alpha, \beta)$ be a candidate Gram matrix of order $n \geq d+1$. Let $\overline{\mathscr{M}}$ denote the set of all $(d+1) \times(d+1)$ submatrices of $C$. Let $\omega$ be an auxiliary variable. If the following system of polynomial equations,

$$
\left\{\begin{array}{l}
\operatorname{det} M(\alpha, \beta)=0 \quad \text { for all } M \in \mathscr{M}, \\
\omega \alpha \beta\left(\alpha^{2}-\beta^{2}\right)\left(\alpha^{2}-1\right)\left(\beta^{2}-1\right)+1=0,
\end{array}\right.
$$

has no solutions in $\mathbb{C}^{3}$, then $\operatorname{rank} C\left(\alpha^{*}, \beta^{*}\right) \leq d$ cannot hold for any $\alpha^{*}, \beta^{*} \in \mathbb{R}$ subject to (2).

Proof Indeed, if rank $C\left(\alpha^{*}, \beta^{*}\right) \leq d$ for some $\alpha^{*}, \beta^{*} \in \mathbb{C}$ subject to (2), then necessarily all $(d+1) \times(d+1)$ minors of $C\left(\alpha^{*}, \beta^{*}\right)$ are vanishing. In particular, there exists an $\omega^{*} \in \mathbb{C}$ such that $\left(\alpha^{*}, \beta^{*}, \omega^{*}\right) \in \mathbb{C}^{3}$ is a solution of the system of equations (3).

We remark that one can decide whether a system of polynomial equations with rational coefficients has any complex solutions by computing a Gröbner basis [6]. 
Based on these concepts, we now may classify biangular line systems in the following way. First, we fix $d \geq 2$ and $n=\left(\begin{array}{c}d+3 \\ 4\end{array}\right)$. Secondly, we generate (by computers, say) all $n \times n$ candidate Gram matrices. Thirdly, for each candidate Gram matrix $C(\alpha, \beta)$ generated, we attempt to determine, via solving the system of equations (3) the (not necessarily finite) set of all real matrices $\left\{C\left(\alpha_{i}^{*}, \beta_{i}^{*}\right): \operatorname{rank} C\left(\alpha_{i}^{*}, \beta_{i}^{*}\right) \leq d, i \in \mathscr{I}\right\}$. Finally, we keep only those which are positive semidefinite. When no such matrices are found, then we decrease $n$ by one and repeat the same procedure.

There are several weak points of this naive method restricting heavily its utility. First of all, the bound on $n$, stipulated by Theorem 3.1, is rather crude, and there is no way to generate all candidate Gram matrices of that size. Secondly, when the solution set of (3) is infinite, then it is a very delicate task to parametrize the matrices $C\left(\alpha_{i}^{*}, \beta_{i}^{*}\right)$, $i \in \mathscr{I}$, and to describe which of these are positive semidefinite. We overcome these difficulties by sophisticated matrix generation techniques, and using Proposition 3.5 for discarding a large fraction of small candidate Gram matrices. We discuss these efforts in the next subsection.

\subsection{The Framework in Detail}

In this subsection we describe in more detail how to generate candidate Gram matrices in an equivalence-free exhaustive manner. The main technical tool is canonization, see [30, Section 4.2.2] and [42]. The vectorization of a candidate Gram matrix $C$ of order $n$ is the vector vec $C:=\left[C_{21}, C_{31}, C_{32}, \ldots, C_{n 1}, \ldots, C_{n, n-1}\right]$. We say that a candidate Gram matrix $C(\alpha, \beta)$ is in canonical form, if it holds that

$$
\begin{array}{r}
\operatorname{vec} C(\alpha, \beta):=\min \left\{\operatorname{vec}\left(P C(\sigma(\alpha), \sigma(\beta)) P^{T}\right): P\right. \text { is a signed } \\
\text { permutation matrix, } \sigma \text { is a relabeling }\},
\end{array}
$$

where comparison of vectors is done lexicographically (one may assume, e.g., that the entries are ordered as $0 \prec \alpha \prec-\alpha \prec \beta \prec-\beta$ ). One particularly attractive feature of the above canonical form is that the leading principal submatrices of canonical matrices are themselves canonical. Therefore canonical matrices can be generated inductively, using smaller canonical matrices as 'seeds'. This method is usually called 'orderly generation'.

Lemma 3.6 The number of $n \times n$ canonical candidate Gram matrices with entries $\{0, \pm \alpha, \pm \beta\}$ (in which all three symbols do not appear simultaneously) is given in Table 3 for $n \in\{1, \ldots, 8\}$.

Proof Case $n=1$ is [1], case $n=2$ are

$$
\left[\begin{array}{ll}
1 & 0 \\
0 & 1
\end{array}\right] \text { and }\left[\begin{array}{ll}
1 & \alpha \\
\alpha & 1
\end{array}\right] \text {. }
$$

Table 3 The number of candidate Gram matrices up to equivalence

\begin{tabular}{lllllllll}
\hline$n$ & 1 & 2 & 3 & 4 & 5 & 6 & 7 & 8 \\
\hline$\#$ & 1 & 2 & 5 & 25 & 194 & 7958 & 1818859 & 1773789830 \\
\hline
\end{tabular}


Case $n=3$ is shown in Example 3.3. The remaining cases follow by computation.

As seen from Table 3, the number of $n \times n$ candidate Gram matrices grows very rapidly. However, when $d \geq 2$ is fixed and $n=d+2$, then we may filter out a very large fraction of candidate Gram matrices with the aid of Proposition 3.5. Indeed, for a given candidate Gram matrix we can check whether (3) has any complex solutions by computing a degree reverse lexicographic reduced Gröbner basis [6], and keep only those candidate Gram matrices in a set $\mathscr{C}_{d}(n)$ for which some solutions are found. We performed this step with the aid of the $\mathrm{C}++$ library 'CoCoA' [1].

We proceed by augmenting each candidate Gram matrix $C \in \mathscr{C}_{d}(n)$ with a new row (and column) whose prefix $\left[C_{n+1,1}, C_{n+1,2}, \ldots, C_{n+1, n-1}\right]$ is lexicographically larger than the respective prefix of the last row of $C$ (cf. (4)), keeping only those canonical matrices which in addition survive the next computationally cheap test.

Lemma 3.7 (combinatorial test) Let $d \geq 2$ be fixed and let $\mathscr{C}_{d}(n)$ be a set containing all pairwise inequivalent candidate Gram matrices of order $n$ for which the system of equations (3) has a solution. Let $C$ be a candidate Gram matrix of order $n+1$. If $C$ corresponds to a Gram matrix in $\mathbb{R}^{d}$, then necessarily all its $n+1$ principal submatrix of order $n$ belong to the set $\mathscr{C}_{d}(n)$, up to equivalence.

Proof Indeed, if $C$ corresponds to some Gram matrix, then there exist real numbers $\alpha^{*}, \beta^{*}$ (subject to (2)) such that rank $C\left(\alpha^{*}, \beta^{*}\right) \leq d$. Since the rank of submatrices cannot increase, this must be true for every principal submatrix of $C\left(\alpha^{*}, \beta^{*}\right)$. Therefore they must be in the set $\mathscr{C}_{d}(n)$, up to equivalence.

Since the $n \times n$ principal submatrix of a candidate Gram matrix of order $n+1$ must be compatible, we test them further with the following.

Corollary 3.8 (weak Gröbner test, cf. Proposition 3.5) Let $d \geq 2$ be fixed, and let $C(\alpha, \beta)$ be a candidate Gram matrix of order $n \geq d+1$. Let $\mathscr{M}$ denote the set of all $(d+1) \times(d+1)$ principal submatrices of $C$. Let $\omega$ be an auxiliary variable. If the following system of polynomial equations,

$$
\left\{\begin{array}{l}
\operatorname{det} M(\alpha, \beta)=0, \quad \text { for all } M \in \mathscr{M}, \\
\omega \alpha \beta\left(\alpha^{2}-\beta^{2}\right)\left(\alpha^{2}-1\right)\left(\beta^{2}-1\right)+1=0,
\end{array}\right.
$$

has no solutions in $\mathbb{C}^{3}$, then $\operatorname{rank} C\left(\alpha^{*}, \beta^{*}\right) \leq d$ cannot hold for any $\alpha^{*}, \beta^{*} \in \mathbb{R}$ subject to (2).

Proof This is a variant of Proposition 3.5.

Finally, we store all surviving matrices in a set $\mathscr{C}_{d}(n+1)$, and repeat this procedure as long as new matrices are discovered (but until $n$ reaches the absolute bound from Theorem 3.1). Once the largest candidate Gram matrices are found, we use Proposition 3.5 to determine explicitly the matrices with rank at most $d$, and then by computing their characteristic polynomial (or eigenvalues, if it is possible) we determine the positive semidefinite matrices. We remark that the set of inner products of the maximum Gram matrices is a by-product of this procedure. We summarize our approach in the following 'roadmap' which we will frequently use as a convenient reference. 
Roadmap 3.9 The following is our approach for generating and classifying biangular lines in $\mathbb{R}^{d}$ :

- Fix the dimension $d \geq 2$.

- Generate all $\{0, \pm \alpha, \pm \beta\}$ canonical candidate Gram matrices (with at most two symbols) of size $d+1$, and store them in a set $\mathscr{C}_{d}(d+1)$.

- Augment every $C \in \mathscr{C}_{d}(d+1)$ with a new row and column in every possible way, and then test the canonical matrices by Proposition 3.5. Store the surviving matrices of size $d+2$ in a set $\mathscr{C}_{d}(d+2)$.

- For every $i \in\left\{d+2, \ldots,\left(\begin{array}{c}d+3 \\ 4\end{array}\right)\right\}$ augment every $C \in \mathscr{C}_{d}(i)$ with a new row and column in every possible way, and then test the canonical matrices by Lemma 3.7 and Corollary 3.8. Store the surviving matrices of size $i+1$ in a set $\mathscr{C}_{d}(i+1)$, and repeat this step.

- For the largest candidate Gram matrices use Proposition 3.5 and in particular the solutions of the system of equations (3) to determine the real matrices of rank at most $d$.

- Select from these the positive semidefinite matrices.

Remark 3.10 We observed that once the size $n$ of candidate Gram matrices is large enough, say $n \geq d+5$, then essentially all matrices survive Corollary 3.8. In these cases we solely rely on Lemma 3.7 for pruning. We believe that the reason for this phenomenon is related to the fact that the congruence order of $\mathbb{R}^{d}$ is $d+3$, see [34, Thm. 7.2].

Remark 3.11 Let $d \geq 3, n \geq d+1, \alpha^{*}, \beta^{*} \in \mathbb{R}$ fixed, and let $C\left(\alpha^{*}, \beta^{*}\right)$ be an $n \times n$ Gram matrix with rank $C\left(\alpha^{*}, \beta^{*}\right) \leq d-2$. Then for every $v \in \mathbb{R}^{n}$,

$$
\operatorname{rank}\left[\begin{array}{cc}
C\left(\alpha^{*}, \beta^{*}\right) & v^{T} \\
v & 1
\end{array}\right] \leq d
$$

by subadditivity. In particular, the tests described in Proposition 3.5 and Corollary 3.8 have no effect.

Remark 3.12 There are two major techniques for matrix canonization: one relies on formula (4) which nicely fits into the framework of 'orderly generation'. The other possibility is to transform the problem of matrix canonization to graph canonization for which there are readily available efficient implementations, such as the 'nauty' software [35]. In Appendix A we describe a graph representation of candidate Gram matrices, which can be used in the framework of 'canonical augmentation'. These two techniques are of similar efficiency, and we have used both of them to cross-check our results. We refer the reader to [11] and references therein.

\section{Classification of Maximum Biangular Lines}

We implemented the framework developed in Sect. 3 in $\mathrm{C}++$ and used a computer cluster with $500 \mathrm{CPU}$ cores for several weeks to obtain the following new classification 
Table $4\{0, \pm \alpha, \pm \beta\}$ candidate Gram matrices in $\mathbb{R}^{2}$

\begin{tabular}{llllll}
\hline$n$ & 2 & 3 & 4 & 5 & 6 \\
\hline$\left|\mathscr{C}_{2}(n)\right|$ & 2 & 3 & 2 & 1 & 0 \\
\hline
\end{tabular}

results in $\mathbb{R}^{d}$ for $d \leq 6$. For completeness, we begin our discussion with the case $d=2$ by giving an independent, computational proof to Lemma 2.1.

Lemma 4.1 (equivalent restatement of Lemma 2.1) The maximum cardinality of a biangular line system in $\mathbb{R}^{2}$ is 5 . The unique configuration has candidate Gram matrix

$$
C(\alpha, \beta)=\left[\begin{array}{lllll}
1 & \alpha & \alpha & \beta & \beta \\
\alpha & 1 & \beta & \alpha & \beta \\
\alpha & \beta & 1 & \beta & \alpha \\
\beta & \alpha & \beta & 1 & \alpha \\
\beta & \beta & \alpha & \alpha & 1
\end{array}\right]
$$

and Gram matrix $C((\sqrt{5}-1) / 4,(-\sqrt{5}-1) / 4)$, describing the main diagonals of the convex regular decagon.

Proof The proof follows Roadmap 3.9 with $d=2$. In Table 4 we display the number of surviving candidate Gram matrices, that is, the numbers $\left|\mathscr{C}_{2}(n)\right|$ for $n \in\{2, \ldots, 6\}$. Since $\left|\mathscr{C}_{2}(6)\right|=0$, it follows that $\left|\mathscr{C}_{2}(n)\right|=0$ for every $n \geq 6$. The unique maximum candidate Gram matrix of size 5 is shown in (5) from which the Gram matrices can be recovered by solving the system of equations (3). It follows that $4 \alpha^{2}+2 \alpha-1=0$ and $\beta=-\alpha-1 / 2$. This yields two permutation equivalent, positive semidefinite solutions: $C((\sqrt{5}-1) / 4,(-\sqrt{5}-1) / 4)$ and $C((-\sqrt{5}-1) / 4,(\sqrt{5}-1) / 4)$, both corresponding to the main diagonals of the convex regular decagon.

Remark 4.2 The four lines, passing through the antipodal vertices of the convex regular octagon form the second largest, inextendible configuration of biangular lines in $\mathbb{R}^{2}$ with the set of inner products $\{0, \pm 1 / \sqrt{2}\}$.

Theorem 4.3 The maximum cardinality of a biangular line system in $\mathbb{R}^{3}$ is 10 . The unique configuration has candidate Gram matrix

$$
C(\alpha, \beta)=\left[\begin{array}{cccccccccc}
1 & \alpha & \alpha & \alpha & \alpha & \alpha & \alpha & \beta & \beta & \beta \\
\alpha & 1 & \alpha & -\alpha & -\alpha & \beta & -\beta & \alpha & -\alpha & \beta \\
\alpha & \alpha & 1 & \beta & -\beta & -\alpha & -\alpha & -\alpha & \alpha & \beta \\
\alpha & -\alpha & \beta & 1 & -\alpha & -\beta & \alpha & -\alpha & \beta & \alpha \\
\alpha & -\alpha & -\beta & -\alpha & 1 & \alpha & \beta & \beta & \alpha & -\alpha \\
\alpha & \beta & -\alpha & -\beta & \alpha & 1 & -\alpha & \beta & -\alpha & \alpha \\
\alpha & -\beta & -\alpha & \alpha & \beta & -\alpha & 1 & \alpha & \beta & -\alpha \\
\beta & \alpha & -\alpha & -\alpha & \beta & \beta & \alpha & 1 & \alpha & \alpha \\
\beta & -\alpha & \alpha & \beta & \alpha & -\alpha & \beta & \alpha & 1 & \alpha \\
\beta & \beta & \beta & \alpha & -\alpha & \alpha & -\alpha & \alpha & \alpha & 1
\end{array}\right]
$$

and Gram matrix $C(1 / 3, \sqrt{5} / 3)$, corresponding to the main diagonals of the platonic dodecahedron. 
Table $5\{0, \pm \alpha, \pm \beta\}$ candidate Gram matrices in $\mathbb{R}^{3}$

\begin{tabular}{lllllllllll}
\hline$n$ & 2 & 3 & 4 & 5 & 6 & 7 & 8 & 9 & 10 & 11 \\
\hline$\left|\mathscr{C}_{3}(n)\right|$ & 2 & 5 & 22 & 23 & 12 & 5 & 2 & 1 & 1 & 0 \\
\hline
\end{tabular}

Table $6\{0, \pm \alpha, \pm \beta\}$ candidate Gram matrices in $\mathbb{R}^{4}$

\begin{tabular}{lllllllllllll}
\hline$n$ & 2 & 3 & 4 & 5 & 6 & 7 & 8 & 9 & 10 & 11 & 12 & 13 \\
\hline$\left|\mathscr{C}_{4}(n)\right|$ & 2 & 5 & 25 & 191 & 701 & 184 & 69 & 27 & 14 & 3 & 3 & 0 \\
\hline
\end{tabular}

Proof The proof follows Roadmap 3.9 with $d=3$. In Table 5 we display the number of surviving candidate Gram matrices, that is, the numbers $\left|\mathscr{C}_{3}(n)\right|$ for $n \in\{2, \ldots, 11\}$. Since $\left|\mathscr{C}_{3}(11)\right|=0$, it follows that $\left|\mathscr{C}_{3}(n)\right|=0$ for every $n \geq 11$. The unique maximum candidate Gram matrix of size 10 is shown in (6). Equations (3) imply that $\alpha=1 / 3$ and $\beta^{2}=5 / 9$. This yields two permutation equivalent, positive semidefinite solutions: $C(1 / 3, \sqrt{5} / 3)$ and $C(1 / 3,-\sqrt{5} / 3)$, both corresponding to the main diagonals of the platonic dodecahedron.

Remark 4.4 The second largest among inextendible examples in $\mathbb{R}^{3}$ can be obtained by lifting the convex regular heptagon by Proposition 2.6 to two carefully chosen heights.

Theorem 4.5 The maximum cardinality of a biangular line system in $\mathbb{R}^{4}$ is 12 . There are four pairwise nonisometric maximum configurations: the shortest vectors of the $D_{4}$ lattice; the shortest vectors of the $\mathrm{D}_{3}$ lattice after lifting; and two spherical 3-distance sets with the common candidate Gram matrix

$$
C(\alpha, \beta)=\left[\begin{array}{cc}
B(\alpha, \beta)+I_{6} & B(\beta, \alpha)-\beta I_{6} \\
B(\beta, \alpha)-\beta I_{6} & B(\alpha, \beta)+I_{6}
\end{array}\right], \quad B(\alpha, \beta)=\left[\begin{array}{cccccc}
0 & \alpha & \alpha & \alpha & \alpha & \alpha \\
\alpha & 0 & \alpha & \beta & \beta & \alpha \\
\alpha & \alpha & 0 & \alpha & \beta & \beta \\
\alpha & \beta & \alpha & 0 & \alpha & \beta \\
\alpha & \beta & \beta & \alpha & 0 & \alpha \\
\alpha & \alpha & \beta & \beta & \alpha & 0
\end{array}\right]
$$

yielding nonisometric Gram matrices $C((3-2 \sqrt{5}) / 11,(4+\sqrt{5}) / 11)$ and $C((3+$ $2 \sqrt{5}) / 11,(4-\sqrt{5}) / 11)$.

Proof The proof follows Roadmap 3.9 with $d=4$. In Table 6 we display the number of surviving candidate Gram matrices, that is, the numbers $\left|\mathscr{C}_{4}(n)\right|$ for $n \in\{2, \ldots, 13\}$. Since $\left|\mathscr{C}_{4}(13)\right|=0$, it follows that $\left|\mathscr{C}_{4}(n)\right|=0$ for every $n \geq 13$. The candidate Gram matrices corresponding to the $D_{4}$ and the lifted $D_{3}$ lattice vectors are not shown here, as they can be easily recovered from Lemma 2.3 and Proposition 2.6, and one may check by solving (3) that these are the only solutions. Interestingly, the third candidate Gram matrix $C(\alpha, \beta)$ shown in (7) yields two nonisometric solutions, as the equations (3) imply that $11 \alpha^{2}-6 \alpha-1=0$ and $\beta=\alpha / 2-1 / 2$. 
Table $7\{0, \pm \alpha, \pm \beta\}$ candidate Gram matrices in $\mathbb{R}^{5}$

\begin{tabular}{llllllllll}
\hline$n$ & $\left|\mathscr{C}_{5}(n)\right|$ & $n$ & $\left|\mathscr{C}_{5}(n)\right|$ & $n$ & $\left|\mathscr{C}_{5}(n)\right|$ & $n$ & $\left|\mathscr{C}_{5}(n)\right|$ & $n$ & $\left|\mathscr{C}_{5}(n)\right|$ \\
\hline 6 & 7954 & 10 & 48448 & 14 & 38826 & 18 & 984 & 22 & 4 \\
7 & 47418 & 11 & 54750 & 15 & 22887 & 19 & 201 & 23 & 1 \\
8 & 27905 & 12 & 56548 & 16 & 10533 & 20 & 45 & 24 & 1 \\
9 & 37381 & 13 & 52246 & 17 & 3701 & 21 & 10 & 25 & 0 \\
\hline
\end{tabular}

Table $8\{0, \pm \alpha, \pm \beta\}$ candidate Gram matrices in $\mathbb{R}^{6}$

\begin{tabular}{llllllllll}
\hline$n$ & $\left|\mathscr{C}_{6}(n)\right|$ & $n$ & $\left|\mathscr{C}_{6}(n)\right|$ & $n$ & $\left|\mathscr{C}_{6}(n)\right|$ & $n$ & $\left|\mathscr{C}_{6}(n)\right|$ & $n$ & $\left|\mathscr{C}_{6}(n)\right|$ \\
\hline 8 & 6883459 & 15 & 11810513 & 22 & 30226589 & 29 & 646252 & 36 & 85 \\
9 & 3170550 & 16 & 17409677 & 23 & 23679948 & 30 & 243144 & 37 & 18 \\
10 & 4107292 & 17 & 24048177 & 24 & 16808810 & 31 & 81562 & 38 & 5 \\
11 & 5260036 & 18 & 30449143 & 25 & 10794327 & 32 & 24461 & 39 & 1 \\
12 & 5781148 & 19 & 35103515 & 26 & 6260018 & 33 & 6554 & 40 & 1 \\
13 & 6239734 & 20 & 36779026 & 27 & 3270750 & 34 & 1610 & 41 & 0 \\
\hline
\end{tabular}

We note that since the candidate Gram matrix (7) describes a spherical 3-distance set, it has already been generated earlier in [44].

Remark 4.6 The Gram matrices obtained from (7) are contained in the Bose-Mesner algebra of a 3-class association scheme [25].

Theorem 4.7 The maximum cardinality of a biangular line system in $\mathbb{R}^{5}$ is 24 . The unique configuration can be obtained by lifting the shortest vectors of the $D_{4}$ lattice.

Proof The proof follows Roadmap 3.9 with $d=5$. In Table 7 we display the number of surviving candidate Gram matrices, that is, the numbers $\left|\mathscr{C}_{5}(n)\right|$ for $n \in\{6, \ldots, 25\}$. Since $\left|\mathscr{C}_{5}(25)\right|=0$, it follows that $\left|\mathscr{C}_{5}(n)\right|=0$ for every $n \geq 25$. The candidate Gram matrix corresponding to the lifted $D_{4}$ lattice vectors is not shown here, as it can be easily recovered from Lemma 2.3 and Proposition 2.6, and one may check by solving (3) that it is the only maximum solution.

Remark 4.8 We remark that the Bose-Mesner algebra (see [25]) of a particular example of 4-class association schemes on 24 vertices contains the maximum Gram matrix $G$ of biangular lines in $\mathbb{R}^{5}$, up to equivalence. Furthermore, since $G^{2}=(24 / 5) G, G$ is a sporadic example of biangular tight frames [14].

The main computational result of this paper is the following.

Theorem 4.9 The maximum cardinality of a biangular line system in $\mathbb{R}^{6}$ is 40 . The unique configuration can be obtained by lifting the shortest vectors of the $D_{5}$ lattice. 
Proof The proof follows Roadmap 3.9 with $d=6$. In Table 8 we display the number of surviving candidate Gram matrices, that is, the numbers $\left|\mathscr{C}_{6}(n)\right|$ for $n \in\{8, \ldots, 41\}$. Since $\left|\mathscr{C}_{6}(41)\right|=0$, it follows that $\left|\mathscr{C}_{6}(n)\right|=0$ for every $n \geq 41$. The candidate Gram matrix corresponding to the lifted $D_{5}$ lattice vectors is not shown here, as it can be easily recovered from Lemma 2.3 and Proposition 2.6, and one may check by solving (3) that it is the only maximum solution.

In dimension 5 and 6 the largest biangular line systems with irrational angles consist of 20 and 24 lines respectively, each having exactly the same inner product set $\{ \pm(3-$ $2 \sqrt{5}) / 11, \pm(4+\sqrt{5}) / 11\}$ as one of the largest configurations in $\mathbb{R}^{4}$ (cf. Theorem 4.5). Examples of these are shown in Appendix B.

Remark 4.10 In $\mathbb{R}^{6}$ two $27 \times 27$ candidate Gram matrices were found corresponding to Gram matrices with angle set $\{ \pm 1 / 4, \pm 1 / 2\}$. It turns out, one of these is the largest spherical 2-distance set [34,37], and the other one belongs to the Bose-Mesner algebra of a 4-class association scheme [25]. See Appendix B.

We note the following by-products of our classification.

Corollary 4.11 The largest infinite family of biangular lines in $\mathbb{R}^{d}$ for $d \in\{3,4,5,6\}$ is formed by 6, 6, 10, and 16 lines, respectively.

Proof For $d=3$ we have the twisted icosahedron [14]. For $d \geq 4$, we can use Proposition 2.5 and well-known spherical 2-distance sets (see [34,37], Examples 3.4 and B.3) in $\mathbb{R}^{d-1}$ to establish the claimed lower bounds. To see that these are indeed the largest, one should inspect the candidate Gram matrices we generated. It is easy to see that if $C(\alpha, \beta)$ is a parametric family of biangular line systems, then the values of $\alpha$ and $\beta$ are not uniquely determined by any of its subsystems. Therefore it is enough to augment those (rather few) candidate Gram matrices for which the dimension of the ideal generated by (3) is positive (see [6]).

Corollary 4.12 The biangular line systems meeting the relative bound in dimension $d \in\{3,4,5,6\}$ for $\alpha^{2}+\beta^{2}<6 /(d+4)$ are exactly those listed in Table 2.

Proof Let $\mathscr{X} \subset \mathbb{R}^{d}$ span a biangular line system meeting the relative bound (1). Since $\alpha^{2}+\beta^{2}<6 /(d+4)$, we have

$$
\sum_{x, x^{\prime} \in \mathscr{X}} C_{2}^{((d-2) / 2)}\left(\left\langle x, x^{\prime}\right\rangle\right)=0 \quad \text { and } \quad \sum_{x, x^{\prime} \in \mathscr{X}} C_{4}^{((d-2) / 2)}\left(\left\langle x, x^{\prime}\right\rangle\right)=0 .
$$

In particular, the antipodal double $\mathscr{Y}:=\{x: x \in \mathscr{X}\} \cup\{-x: x \in \mathscr{X}\}$ is a spherical 5-design [4,10], and hence $|\mathscr{X}|=|\mathscr{Y}| / 2 \geq d(d+1) / 2$. For $d=3$ the only tight spherical 5-design is the icosahedron [4], [20, Exam. 5.16]. For $d \geq 4$ it follows from Corollary 4.11 that the number of Gram matrices of size $|\mathscr{X}|$ is finite, therefore one may plug in the (finitely many) inner products $\alpha^{*}$ and $\beta^{*}$ into (1) to test equality. This yields Table 2 for $d \leq 6$. 
Remark 4.13 If $\alpha^{2}+\beta^{2}=6 /(d+4)$ and there is equality in the relative bound (1), then necessarily

$$
\frac{d^{2}+3|\mathscr{X}|-4}{(d+2)(d+4)}=(|\mathscr{X}|-1) \beta^{2}\left(\frac{6}{d+4}-\beta^{2}\right) \text {. }
$$

For fixed $d$ and $|\mathscr{X}|$ this in turn determines the possible inner products in $A(\mathscr{X})$. Then one may go through all candidate Gram matrices and check which of these inner products are compatible with the solutions of (3). Since we tend to believe that for $d \leq 6$ there are no biangular lines of this type, we have not gone through the details of this lengthy and seemingly very tedious task.

\section{Results on Multiangular Lines}

The theory developed in Sect. 3 can be generalized to multiangular lines in a straightforward manner. The main challenge in our study is solving (the multiangular analogue of) the system of equations (3). Indeed, the efficiency of computing a Gröbner basis very much depends on the number of variables [6], and 4-angular line systems are the largest ones our methods can currently handle. In this section we briefly report on our computational results on multiangular lines.

\subsection{Multiangular Lines in $\mathbb{R}^{3}$}

It is well known that in $\mathbb{R}^{3}$ the main diagonals of the platonic icosahedron form the largest equiangular line system, and we showed in Theorem 4.3 that the main diagonals of the platonic dodecahedron form the largest biangular line system. It is natural to ask what are the multiangular analogues of these objects. On the plane the maximum cardinality of $m$-angular lines is $2 m+1$, and an example is coming from the main diagonals of the convex regular $(4 m+2)$-gon [37].

Theorem 5.1 The maximum cardinality of a triangular line system in $\mathbb{R}^{3}$ is 12 . There are exactly two such configurations coming from the following candidate Gram matrix:

$$
C(\alpha, \beta, \gamma)=\left[\begin{array}{cccccccccccc}
1 & \alpha & \alpha & \alpha & \alpha & \beta & \beta & \beta & \beta & \gamma & \gamma & \gamma \\
\alpha & 1 & \beta & \gamma & \gamma & \alpha & \beta & \beta & \gamma & \alpha & \alpha & \beta \\
\alpha & \beta & 1 & \gamma & -\alpha & \gamma & -\beta & -\gamma & \alpha & \beta & -\beta & \alpha \\
\alpha & \gamma & \gamma & 1 & \beta & \beta & \alpha & \gamma & \beta & \alpha & \beta & \alpha \\
\alpha & \gamma & -\alpha & \beta & 1 & -\beta & \gamma & \alpha & -\gamma & \beta & \alpha & -\beta \\
\beta & \alpha & \gamma & \beta & -\beta & 1 & -\gamma & -\beta & \alpha & \gamma & -\alpha & \alpha \\
\beta & \beta & -\beta & \alpha & \gamma & -\gamma & 1 & \alpha & -\beta & \gamma & \alpha & -\alpha \\
\beta & \beta & -\gamma & \gamma & \alpha & -\beta & \alpha & 1 & -\alpha & \alpha & \gamma & -\beta \\
\beta & \gamma & \alpha & \beta & -\gamma & \alpha & -\beta & -\alpha & 1 & \alpha & -\beta & \gamma \\
\gamma & \alpha & \beta & \alpha & \beta & \gamma & \gamma & \alpha & \alpha & 1 & \beta & \beta \\
\gamma & \alpha & -\beta & \beta & \alpha & -\alpha & \alpha & \gamma & -\beta & \beta & 1 & -\gamma \\
\gamma & \beta & \alpha & \alpha & -\beta & \alpha & -\alpha & -\beta & \gamma & \beta & -\gamma & 1
\end{array}\right],
$$


Table $9\{0, \pm \alpha, \pm \beta, \pm \gamma\}$ candidate Gram matrices in $\mathbb{R}^{3}$

\begin{tabular}{lllllllllllll}
\hline$n$ & 2 & 3 & 4 & 5 & 6 & 7 & 8 & 9 & 10 & 11 & 12 & 13 \\
\hline$\left|\mathscr{C}_{3}(n)\right|$ & 2 & 7 & 62 & 610 & 271 & 104 & 46 & 19 & 6 & 1 & 1 & 0 \\
\hline
\end{tabular}

Table $10\{0, \pm \alpha, \pm \beta, \pm \gamma, \pm \delta\}$ candidate Gram matrices in $\mathbb{R}^{3}$

\begin{tabular}{llllllllll}
\hline$n$ & $\left|\mathscr{C}_{3}(n)\right|$ & $n$ & $\left|\mathscr{C}_{3}(n)\right|$ & $n$ & $\left|\mathscr{C}_{3}(n)\right|$ & $n$ & $\left|\mathscr{C}_{3}(n)\right|$ & $n$ & $\left|\mathscr{C}_{3}(n)\right|$ \\
\hline 2 & 2 & 5 & 7014 & 8 & 632 & 11 & 32 & 14 & 1 \\
3 & 7 & 6 & 7744 & 9 & 276 & 12 & 14 & 15 & 1 \\
4 & 97 & 7 & 1655 & 10 & 104 & 13 & 3 & 16 & 0 \\
\hline
\end{tabular}

namely $C((-7+4 \sqrt{2}) / 17,(5+2 \sqrt{2}) / 17,(-3-8 \sqrt{2}) / 17)$ is the truncated cube and $C((-7-4 \sqrt{2}) / 17,(5-2 \sqrt{2}) / 17,(-3+8 \sqrt{2}) / 17)$ is the small rhombicuboctahedron.

Proof The proof follows analogously to Roadmap 3.9 with $d=3$. In Table 9 we display the number of surviving candidate Gram matrices with symbols $\{0, \pm \alpha, \pm \beta, \pm \gamma\}$ (where at most three out of these four symbols appear), that is, the numbers $\left|\mathscr{C}_{3}(n)\right|$ for $n \in\{2, \ldots, 13\}$. Since $\left|\mathscr{C}_{3}(13)\right|=0$, it follows that $\left|\mathscr{C}_{3}(n)\right|=0$ for every $n \geq 13$. In addition, there is a unique maximum candidate Gram matrix of size 12, as shown in (8). Equations analogous to (3) imply the claimed solutions.

Theorem 5.2 The maximum cardinality of a 4-angular line system in $\mathbb{R}^{3}$ is 15 . There is a unique configuration coming from the following candidate Gram matrix:

$$
C(\alpha, \beta, \gamma)=\left[\begin{array}{ccccccccccccccc}
1 & 0 & 0 & \alpha & \beta & \gamma & \alpha & \beta & \gamma & \alpha & \beta & \gamma & \alpha & \beta & \gamma \\
0 & 1 & 0 & \beta & \gamma & \alpha & \beta & \gamma & \alpha & -\beta & -\gamma & -\alpha & -\beta & -\gamma & -\alpha \\
0 & 0 & 1 & \gamma & \alpha & \beta & -\gamma & -\alpha & -\beta & \gamma & \alpha & \beta & -\gamma & -\alpha & -\beta \\
\alpha & \beta & \gamma & 1 & 0 & 0 & \gamma & -\alpha & -\beta & \alpha & -\beta & \gamma & -\beta & -\gamma & \alpha \\
\beta & \gamma & \alpha & 0 & 1 & 0 & -\alpha & \beta & \gamma & -\beta & \gamma & -\alpha & -\gamma & -\alpha & \beta \\
\gamma & \alpha & \beta & 0 & 0 & 1 & -\beta & \gamma & \alpha & \gamma & -\alpha & \beta & \alpha & \beta & -\gamma \\
\alpha & \beta & -\gamma & \gamma & -\alpha & -\beta & 1 & 0 & 0 & -\beta & -\gamma & \alpha & \alpha & -\beta & \gamma \\
\beta & \gamma & -\alpha & -\alpha & \beta & \gamma & 0 & 1 & 0 & -\gamma & -\alpha & \beta & -\beta & \gamma & -\alpha \\
\gamma & \alpha & -\beta & -\beta & \gamma & \alpha & 0 & 0 & 1 & \alpha & \beta & -\gamma & \gamma & -\alpha & \beta \\
\alpha & -\beta & \gamma & \alpha & -\beta & \gamma & -\beta & -\gamma & \alpha & 1 & 0 & 0 & \gamma & -\alpha & -\beta \\
\beta & -\gamma & \alpha & -\beta & \gamma & -\alpha & -\gamma & -\alpha & \beta & 0 & 1 & 0 & -\alpha & \beta & \gamma \\
\gamma & -\alpha & \beta & \gamma & -\alpha & \beta & \alpha & \beta & -\gamma & 0 & 0 & 1 & -\beta & \gamma & \alpha \\
\alpha & -\beta & -\gamma & -\beta & -\gamma & \alpha & \alpha & -\beta & \gamma & \gamma & -\alpha & -\beta & 1 & 0 & 0 \\
\beta & -\gamma & -\alpha & -\gamma & -\alpha & \beta & -\beta & \gamma & -\alpha & -\alpha & \beta & \gamma & 0 & 1 & 0 \\
\gamma & -\alpha & -\beta & \alpha & \beta & -\gamma & \gamma & -\alpha & \beta & -\beta & \gamma & \alpha & 0 & 0 & 1
\end{array}\right],
$$

namely $C((1+\sqrt{5}) / 4,(1-\sqrt{5}) / 4,1 / 2)$ is the icosidodecahedron.

Proof The proof follows analogously to Roadmap 3.9 with $d=3$, with the following noted difference: first we generated all $5 \times 5$ candidate Gram matrices, and used 
Table $11\{0, \pm \alpha, \pm \beta\}$ candidate Gram matrices in $\mathbb{R}^{4}$

\begin{tabular}{llllllllll}
\hline$n$ & $\left|\mathscr{C}_{4}(n)\right|$ & $n$ & $\left|\mathscr{C}_{4}(n)\right|$ & $n$ & $\left|\mathscr{C}_{4}(n)\right|$ & $n$ & $\left|\mathscr{C}_{4}(n)\right|$ & $n$ & $\left|\mathscr{C}_{4}(n)\right|$ \\
\hline 1 & 1 & 6 & 8353 & 11 & 2694 & 16 & 892 & 21 & 10 \\
2 & 2 & 7 & 2746 & 12 & 2919 & 17 & 447 & 22 & 4 \\
3 & 6 & 8 & 1725 & 13 & 2638 & 18 & 214 & 23 & 1 \\
4 & 51 & 9 & 1776 & 14 & 2147 & 19 & 80 & 24 & 1 \\
5 & 1152 & 10 & 2314 & 15 & 1453 & 20 & 34 & 25 & 0 \\
\hline
\end{tabular}

Proposition 3.5 for filtering the $6 \times 6$ (and larger) matrices. In Table 10 we display the number of surviving candidate Gram matrices with symbols $\{0, \pm \alpha, \pm \beta, \pm \gamma, \pm \delta\}$ (where at most four out of these five symbols appear), that is, the numbers $\left|\mathscr{C}_{3}(n)\right|$ for $n \in\{2, \ldots, 16\}$. Since $\left|\mathscr{C}_{3}(16)\right|=0$, it follows that $\left|\mathscr{C}_{3}(n)\right|=0$ for every $n \geq 16$. In addition, there is a unique maximum candidate Gram matrix of size 15, as shown in (9). Equations analogous to (3) imply that $4 \alpha^{2}-2 \alpha-1=0, \beta=1 / 2-\alpha, \gamma=1 / 2$. This yields two equivalent, positive semidefinite solutions, both corresponding to the main diagonals of the icosidodecahedron.

Remark 5.3 It turns out that the icosidodecahedron is the largest 5-angular configuration in $\mathbb{R}^{3}$ containing orthogonal lines. The search is completely analogous to what is described in Theorem 5.2 and its proof.

We refer the reader to [26] for further interesting arrangements in $\mathbb{R}^{3}$.

\subsection{Higher Dimensional Examples}

In this section we report on our computational results on triangular line systems, where one of the three possible inner products is 0 . On the plane, the unique maximum configuration is formed by the main diagonals of the convex regular 12-gon, and in dimension 3 these are once again the main diagonals of the dodecahedron. Both of these results can be concluded from inspecting the matrices we generated for the proof of Theorem 5.1 (see Table 9).

Theorem 5.4 The maximum cardinality of a triangular line system containing orthogonal lines in $\mathbb{R}^{4}$ is 24 . There is a unique configuration spanned by

$$
\begin{array}{r}
\mathscr{X}=\{[1, \pm 1, \pm 1, \pm 1] / 2\} \cup\{[1,0,0,0],[0,1,0,0],[0,0,1,0],[0,0,0,1]\} \\
\cup\{x: x \text { is a permutation of }[ \pm 1, \pm 1,0,0] / \sqrt{2},\langle x,[4,3,2,1]\rangle>0\},
\end{array}
$$

which describes the main diagonals of the 24-cell and its dual.

Proof The proof follows analogously to Roadmap 3.9 with $d=4$. In Table 11 we display the number of surviving candidate Gram matrices with symbols $\{0, \pm \alpha, \pm \beta\}$, that is, the numbers $\left|\mathscr{C}_{4}(n)\right|$ for $n \in\{2, \ldots, 25\}$. Since $\left|\mathscr{C}_{4}(25)\right|=0$, it follows that 
Table $12\{0, \pm \alpha, \pm \beta\}$ candidate Gram matrices in $\mathbb{R}^{5}$

\begin{tabular}{llllllllll}
\hline$n$ & $\left|\mathscr{C}_{5}(n)\right|$ & $n$ & $\left|\mathscr{C}_{5}(n)\right|$ & $n$ & $\left|\mathscr{C}_{5}(n)\right|$ & $n$ & $\left|\mathscr{C}_{5}(n)\right|$ & $n$ & $\left|\mathscr{C}_{5}(n)\right|$ \\
\hline 7 & 1045395 & 14 & 12214161 & 21 & 68512201 & 28 & 2932142 & 35 & 471 \\
8 & 370512 & 15 & 21063583 & 22 & 59177264 & 29 & 1217479 & 36 & 94 \\
9 & 441556 & 16 & 32845898 & 23 & 46323247 & 30 & 449091 & 37 & 18 \\
10 & 724198 & 17 & 46331977 & 24 & 32824635 & 31 & 146385 & 38 & 4 \\
11 & 1422041 & 18 & 59180410 & 25 & 21019703 & 32 & 41984 & 39 & 1 \\
12 & 3076847 & 19 & 68513149 & 26 & 12137301 & 33 & 10565 & 40 & 1 \\
13 & 6412829 & 20 & 71935169 & 27 & 6301866 & 34 & 2357 & 41 & 0 \\
\hline
\end{tabular}

$\left|\mathscr{C}_{4}(n)\right|=0$ for every $n \geq 25$. The unique largest candidate Gram matrix corresponding to this case can be easily recovered from $\mathscr{X}$, and then solving (3) yields two equivalent solutions with the set of inner products $\{0, \pm 1 / 2, \pm 1 / \sqrt{2}\}$.

Remark 5.5 In $\mathbb{R}^{4}$, the second largest inextendible configuration has cardinality 16 , spanned by all permutations of $[ \pm 1, \pm 1, \pm 1,0] / \sqrt{3}$ where the first nonzero entry is positive. The set of inner products of this configuration is $\{0, \pm 1 / 3, \pm 2 / 3\}$.

Theorem 5.6 The maximum cardinality of a triangular line system containing orthogonal lines in $\mathbb{R}^{5}$ is 40 . This unique configuration is spanned by the set $\mathscr{X}$ of all permutations of $[ \pm 1, \pm 1, \pm 1,0,0] / \sqrt{3}$, where the first nonzero entry is positive.

Proof The proof follows analogously to Roadmap 3.9 with $d=5$. In Table 12 we display the number of surviving candidate Gram matrices with symbols $\{0, \pm \alpha, \pm \beta\}$, that is, the numbers $\left|\mathscr{C}_{5}(n)\right|$ for $n \in\{7, \ldots, 41\}$. Since $\left|\mathscr{C}_{5}(41)\right|=0$, it follows that $\left|\mathscr{C}_{5}(n)\right|=0$ for every $n \geq 41$. The unique largest candidate Gram matrix corresponding to this case can be easily recovered from $\mathscr{X}$, and then solving (3) yields a unique solution with the set of inner products $\{0, \pm 1 / 3, \pm 2 / 3\}$.

\section{Open Problems}

We conclude this paper with the following set of problems.

Problem 6.1 (superquadratic lines, see [3]) Let $c, \varepsilon>0$ be fixed. Find a construction of a series of biangular lines $\mathscr{X}_{d} \subset \mathbb{R}^{d}$ such that $\left|\mathscr{X}_{d}\right| \geq c \cdot d^{2+\varepsilon}$ holds for infinitely many $d \geq 1$.

In particular, investigate if Proposition 2.6 can be applied to a suitable series of spherical 3-distance sets.

Problem 6.2 Find a series of spherical 3-distance sets $\mathscr{X}_{d} \subset \mathbb{R}^{d}$ with $A\left(\mathscr{X}_{d}\right) \subseteq$ $\left\{\alpha_{d}, \beta_{d}, \gamma_{d}\right\}$ such that $\alpha_{d}+\beta_{d}<0$ and $\left|\mathscr{X}_{d}\right|$ is superquadratic (in the sense of Problem 6.1).

Problem 6.3 (see [14]) Find a series of biangular tight frames $\mathscr{X}_{d} \subset \mathbb{R}^{d}$ such that $\left|\mathscr{X}_{d}\right|>d^{2}$ for infinitely many $d \geq 1$. 
It is known that the twisted icosahedron [14] forms an infinite family of six biangular lines in $\mathbb{R}^{3}$, which is one line larger compared to what Proposition 2.5 guarantees.

Problem 6.4 (cf. Corollary 4.11) Determine if there exists an infinite family of biangular lines $\mathscr{X}(h) \subset \mathbb{R}^{d}$ such that $|\mathscr{X}(h)|$ is larger than the one described in Proposition 2.5 for some $d \geq 7$.

Problem 6.5 (see [32], cf. Example B.3) Determine if there exists an infinite family of 28 biangular lines $\mathscr{X}(h) \subset \mathbb{R}^{7}$ such that $\mathscr{X}(0)$ spans equiangular lines.

It would be also very interesting to see whether binary codes with four distinct distances lead to improved constructions in $\mathbb{R}^{d}$ for some $d \leq 23$ or possibly beyond.

Problem 6.6 (see Lemma 2.2) For $d \geq 2$ determine the maximum cardinality of binary codes of length $d$ admitting at most four distinct Hamming distances $\left\{\Delta_{1}, \Delta_{2}, d-\Delta_{1}, d-\Delta_{2}\right\}, \Delta_{1}, \Delta_{2} \in\{1, \ldots, d-1\}$.

Problem 6.7 (cf. Theorem 2.18, Remark 4.13) Determine if there exists a set $\mathscr{X} \subset \mathbb{R}^{d}$ spanning biangular lines with $A(\mathscr{X}) \subseteq\{ \pm \alpha, \pm \beta\}$, such that $\alpha^{2}+\beta^{2}=6 /(d+4)$, and there is equality in (1) for some $d \geq 3$.

Problem 6.8 (see [2]) Complement Table 1 by using the semidefinite programming technique to establish (sharp) upper bounds for the maximum cardinality of biangular lines $\mathscr{X} \subset \mathbb{R}^{d}$ with $A(\mathscr{X}) \subseteq\{ \pm 1 / 5, \pm 3 / 5\}$ for $d \leq 23$.

Acknowledgements We thank Prof. Patric Östergård for providing us (essentially unlimited) access to supercomputing resources at the Aalto University.

Funding Open Access funding provided by the Aalto University.

Open Access This article is licensed under a Creative Commons Attribution 4.0 International License, which permits use, sharing, adaptation, distribution and reproduction in any medium or format, as long as you give appropriate credit to the original author(s) and the source, provide a link to the Creative Commons licence, and indicate if changes were made. The images or other third party material in this article are included in the article's Creative Commons licence, unless indicated otherwise in a credit line to the material. If material is not included in the article's Creative Commons licence and your intended use is not permitted by statutory regulation or exceeds the permitted use, you will need to obtain permission directly from the copyright holder. To view a copy of this licence, visit http://creativecommons.org/licenses/by/4.0/.

\section{Appendix A: Graph Representation of Candidate Gram Matrices}

Let $m \geq 1, n \geq 2$ be integers, and consider an $n \times n$ symmetric matrix $C\left(\alpha_{1}, \ldots, \alpha_{m}\right)$ with constant diagonal entries 1 over the ring $\mathbb{Q}\left[\alpha_{1}, \ldots, \alpha_{m}\right]$ and off-diagonal entries $\left\{0, \pm \alpha_{1}, \ldots, \pm \alpha_{m}\right\}$. Analogously to Definition 3.2, two such matrices $C_{1}$ and $C_{2}$ are called equivalent, if

$$
C_{1}\left(\alpha_{1}, \ldots, \alpha_{m}\right)=P C_{2}\left(\sigma\left(\alpha_{1}\right), \ldots, \sigma\left(\alpha_{m}\right)\right) P^{T}
$$

for some signed permutation matrix $P$ and relabeling $\sigma$. A representative of this matrix equivalence class is called a candidate Gram matrix. 
The goal of this section is to construct for every matrix $C\left(\alpha_{1}, \ldots, \alpha_{m}\right)$ of order $n$ a (colored) graph $X\left(C\left(\alpha_{1}, \ldots, \alpha_{m}\right)\right)$ capturing its underlying symmetries and in particular its equivalence class. With this representation, equivalence of matrices $C_{1}$ and $C_{2}$ (over the same symbol set) simply boils down to the isomorphism of the corresponding colored graphs $X\left(C_{1}\right)$ and $X\left(C_{2}\right)$. This latter task can be readily decided by the 'nauty' software [35] in practice. Our graph $X(C)$ has $2 n^{2}+n+2 m$ vertices, and its vertex set $V(X(C))$ is partitioned into the following four distinct (nonempty) color classes:

$$
V(X(C)):=\mathscr{U} \cup \mathscr{V} \cup \mathscr{W} \cup \mathscr{Z}
$$

Here $\mathscr{U}:=\left\{u_{i}: i \in\{1, \ldots, n\}\right\}$ conceptually represents the $n$ lines (in other words, the $n$ rows/columns of the matrix $C$ ). The set $\mathscr{V}:=\left\{v_{i k}: i \in\{1, \ldots, n\}, k \in\right.$ $\{1,2\}\}$ represents the set of antipodal unit vectors (say $\pm x$ ) spanning the lines. The set $\mathscr{W}:=\left\{w_{i j k}: i<j \in\{1, \ldots, n\}, k \in\{1, \ldots, 4\}\right\}$ represents the four possible inner products $\left\langle \pm x, \pm x^{\prime}\right\rangle$ (where $\pm x$ and $\pm x^{\prime}$ are the spanning unit vectors of distinct lines), and finally $\mathscr{Z}=\left\{z_{i k}: i \in\{1, \ldots, m\}, k \in\{1,2\}\right\}$ represents the $2 m$ off-diagonal entries (where for every $i \in\{1, \ldots, m\}$, the vertices $z_{i 1}$ and $z_{i 2}$ correspond to the same symbol $\alpha_{i}$ and its negative, in some order).

The edge set $E(X(C))$ is given by $E(X(C)):=\mathscr{E}_{1} \cup \mathscr{E}_{2} \cup \mathscr{E}_{3} \cup \mathscr{E}_{4} \cup \mathscr{E}_{5}$, where $\mathscr{E}_{1}:=\left\{\left\{u_{i}, v_{i k}\right\}: i \in\{1, \ldots, n\}, k \in\{1,2\}\right\}$ and $\mathscr{E}_{2}:=\left\{\left\{z_{i 1}, z_{i 2}\right\}: i \in\{1, \ldots, m\}\right\}$ describe the edges connecting the elements of $\mathscr{U}$ and $\mathscr{V}$, and the edges within $\mathscr{Z}$, respectively. Furthermore,

$$
\begin{aligned}
\mathscr{E}_{3}:= & \left\{\left\{v_{i 1}, w_{i j 1}\right\},\left\{v_{i 2}, w_{i j 1}\right\},\left\{w_{i j 1}, w_{i j 2}\right\},\left\{w_{i j 2}, w_{i j 3}\right\},\left\{w_{i j 3}, w_{i j 4}\right\},\right. \\
& \left.\left\{v_{j 1}, w_{i j 4}\right\},\left\{v_{j 2}, w_{i j 4}\right\}: i<j \in\{1, \ldots, n\}, G_{i j}=0\right\}
\end{aligned}
$$

and

$$
\begin{aligned}
\mathscr{E}_{4}:= & \left\{\left\{v_{i k}, w_{i j k}\right\},\left\{v_{j k}, w_{i j k}\right\},\left\{v_{i k}, w_{i j(k+2)}\right\},\right. \\
& \left.\left\{v_{j(3-k)}, w_{i j(k+2)}\right\}: i<j \in\{1, \ldots, n\}, k \in\{1,2\}, G_{i j} \neq 0\right\}
\end{aligned}
$$

describe the graph structure between (vertices representing) orthogonal and nonorthogonal lines, respectively. Finally,

$$
\begin{aligned}
\mathscr{E}_{5}:= & \left\{\left\{w_{i j k}, z_{\ell 1}\right\},\left\{w_{i j(k+2)}, z_{\ell 2}\right\}: i<j \in\{1, \ldots, n\}, k \in\{1,2\}, G_{i j}=\alpha_{\ell}\right\} \\
& \cup\left\{\left\{w_{i j k}, z_{\ell 2}\right\},\left\{w_{i j(k+2)}, z_{\ell 1}\right\}: i<j \in\{1, \ldots, n\}, k \in\{1,2\}, G_{i j}=-\alpha_{\ell}\right\}
\end{aligned}
$$

describes the edges connecting the vertices between $\mathscr{W}$ and $\mathscr{Z}$, thus providing a correspondence between lines with certain inner products, and the symbols representing these inner products.

This graph representation is very powerful, as we have the following. 


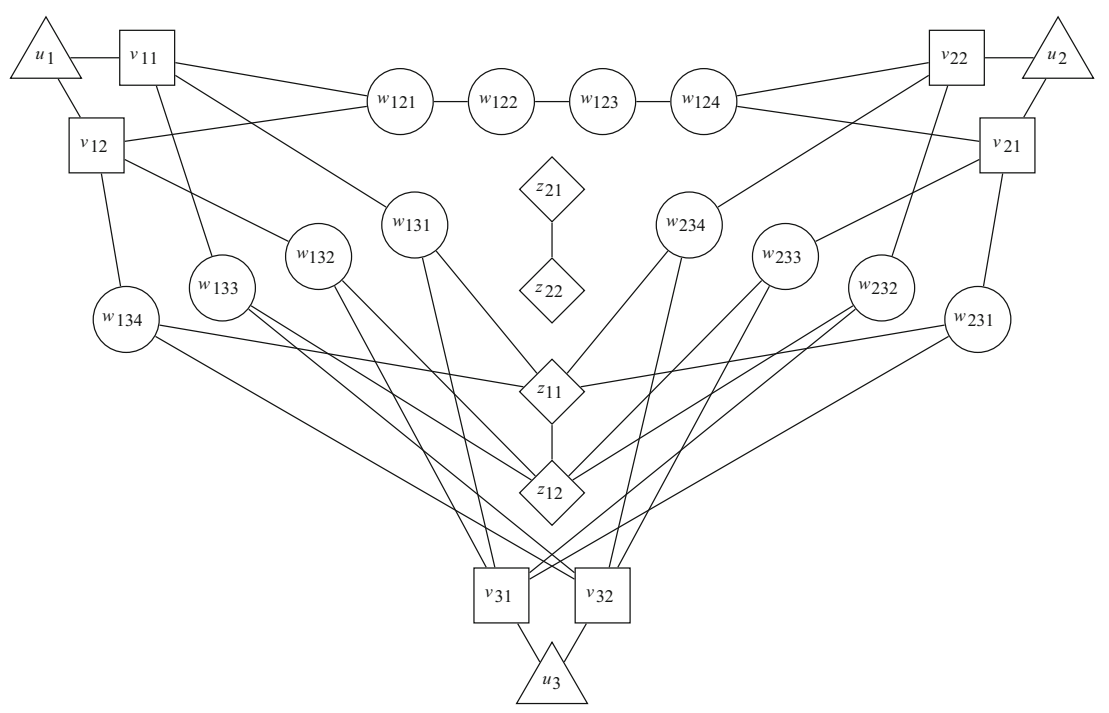

Fig. 1 Graph representation of a candidate Gram matrix

Proposition A.1 The matrices $C_{1}$ and $C_{2}$ (over the same symbol set) are equivalent, if and only if $X\left(C_{1}\right)$ and $X\left(C_{2}\right)$ are isomorphic as graphs. Furthermore, the automorphism groups of $C_{1}$ and $X\left(C_{1}\right)$ are isomorphic as groups.

We omit the proof and refer the reader to [35]. Instead, we show how to represent

$$
\left[\begin{array}{ccc}
1 & 0 & \alpha \\
0 & 1 & \alpha \\
\alpha & \alpha & 1
\end{array}\right]
$$

over the symbol set $\{ \pm \alpha, \pm \beta\}$ in Fig. 1 .

\section{Appendix B: Miscellaneous Matrices}

We note the largest biangular line systems in $\mathbb{R}^{5}$ and $\mathbb{R}^{6}$ containing a pair of lines with irrational inner product between them. It turns out that all of these examples have inner product set $\left\{ \pm \alpha^{*}, \pm \beta^{*}\right\}$, where $\alpha^{*}:=(3-2 \sqrt{5}) / 11, \beta^{*}:=(4+\sqrt{5}) / 11$ are exactly the same values as stated in Theorem 4.5. Furthermore, the two examples shown below are extensions of one of the 12-dimensional maximum cases. Indeed, their upper left $12 \times 12$ submatrix agrees with the matrix shown in (7).

Example B.1 The largest cardinality of a biangular line system in $\mathbb{R}^{5}$ with an irrational inner product is 20 . There are 12 candidate Gram matrices, each corresponding to a single line system. The following candidate Gram matrix (with $\gamma:=-\alpha$ and $\delta:=-\beta$ ) 


\begin{tabular}{|c|c|}
\hline$C(\alpha, \beta, \gamma, \delta)=$ & 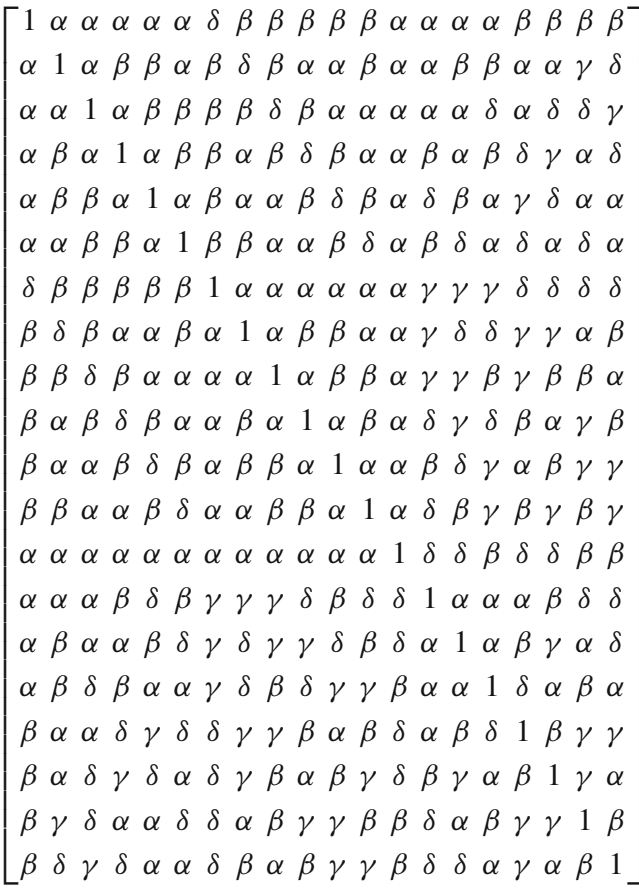 \\
\hline
\end{tabular}

yields a Gram matrix $C\left(\alpha^{*}, \beta^{*},-\alpha^{*},-\beta^{*}\right)$.

Example B.2 The largest biangular line system in $\mathbb{R}^{6}$ with irrational inner products is a unique configuration of 24 lines, corresponding to the candidate Gram matrix (10) (where $\gamma:=-\alpha$ and $\delta:=-\beta$ ). The matrix $C\left(\alpha^{*}, \beta^{*},-\alpha^{*},-\beta^{*}\right)$ is positive semidefinite of rank 6 .

Example B.3 (Schläfli graph) In $\mathbb{R}^{6}$, there is a well-known spherical 2-distance set of cardinality 27 with the set of inner products $\{-1 / 2,1 / 4\}$, related to the adjacency matrix of the Schläfli graph [34,37]. Let $C(\alpha, \beta, \gamma, \delta)$ be as in (11). Then $C(1,0,0,1)-I_{27}$ is the graph adjacency matrix of the Schläfli graph, and $C(1 / 4,-1 / 2,-1 / 2,1 / 4)$ is a spherical two-distance set spanning biangular lines. Application of Proposition 2.5 yields an infinite family of 27 biangular lines in $\mathbb{R}^{7}$. It turns out that $C(1 / 4,-1 / 2,1 / 2,-1 / 4)$ is an additional, nonisometric example in $\mathbb{R}^{6}$, coming from a 4-class association scheme [25]. The antipodal double of this set is a new example of spherical 5-designs [4]. 


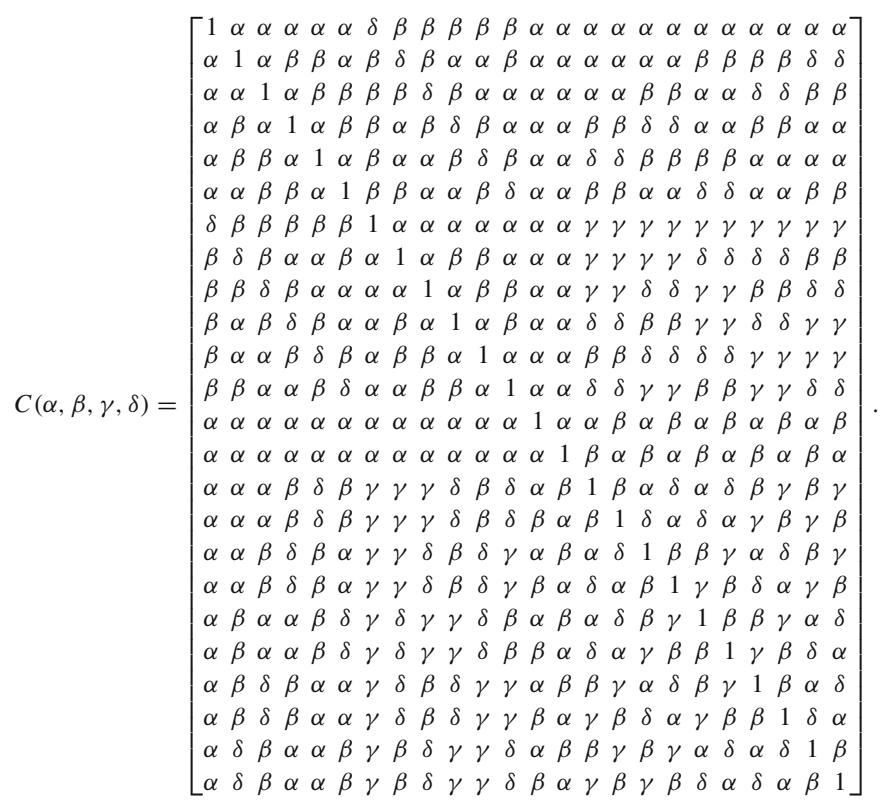

(10)

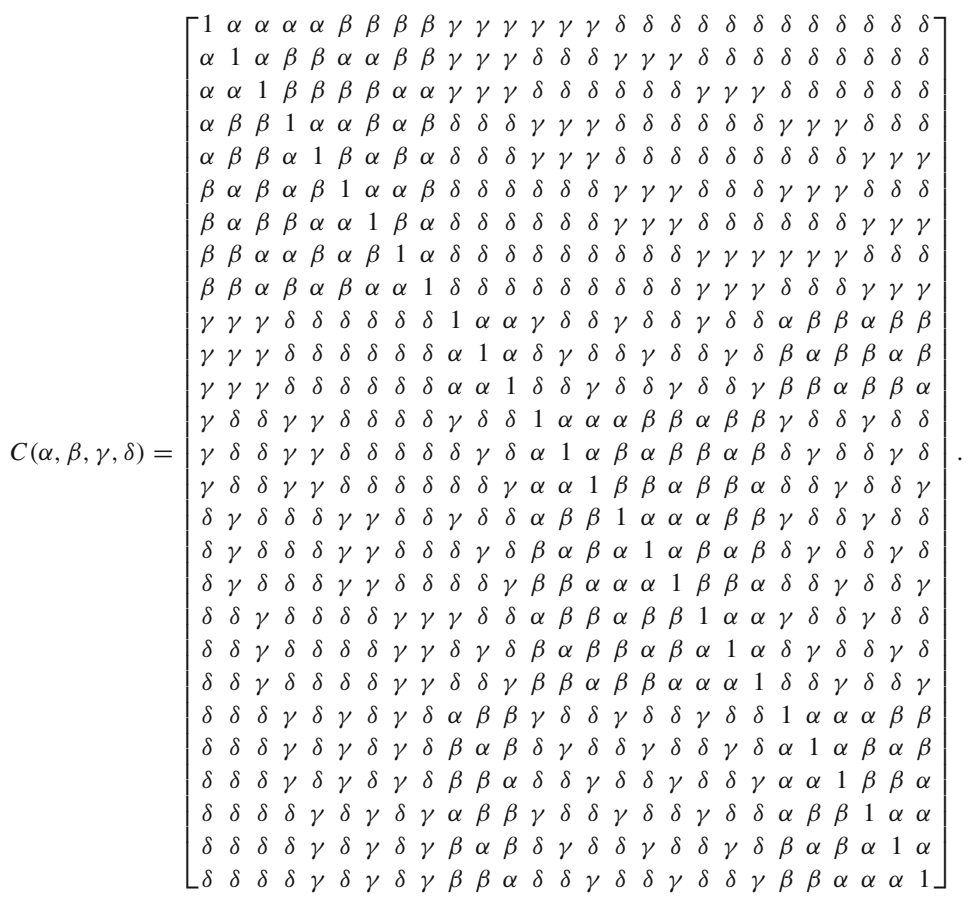




\section{References}

1. Abbott, J., Bigatti, A.M.: CoCoALib: a C++ library for doing Computations in Commutative Algebra. http://cocoa.dima.unige.it/cocoalib, ver. 0.99560 (2019)

2. Bachoc, Ch., Vallentin, F.: Optimality and uniqueness of the $(4,10,1 / 6)$ spherical code. J. Combin. Theory Ser. A 116(1), 195-204 (2009)

3. Balla, I., Dräxler, F., Keevash, P., Sudakov, B.: Equiangular lines and spherical codes in Euclidean space. Invent. Math. 211(1), 179-212 (2018)

4. Bannai, Ei., Bannai, Et.: A survey on spherical designs and algebraic combinatorics on spheres. European J. Combin. 30(6), 1392-1425 (2009)

5. Bannai, Ei., Bannai, Et., Stanton, D.: An upper bound for the cardinality of an $s$-distance subset in real Euclidean space, II. Combinatorica 3(2), 147-152 (1983)

6. Becker, T., Weispfenning, V.: Gröbner Bases. Graduate Texts in Mathematics, vol. 141. Springer, New York (1993)

7. Best, D.: Biangular Vectors. MSc thesis, University of Lethbridge (2013). https://opus.uleth.ca/handle/ $10133 / 3560$

8. Best, D., Kharaghani, H., Ramp, H.: Mutually unbiased weighing matrices. Des. Codes Cryptogr. 76(2), 237-256 (2015)

9. Blokhuis, A.: Few-Distance Sets. CWI Tract, vol. 7. Stichting Mathematisch Centrum, Centrum voor Wiskunde en Informatica, Amsterdam (1984)

10. Boyvalenkov, P., Delchev, K.: On maximal antipodal spherical codes with few distances. Elec. Notes Discr. Math. 57, 85-90 (2017)

11. Brinkmann, G.: Fast generation of cubic graphs. J. Graph Theory 23(2), 139-149 (1996)

12. Brouwer, A.E.: Block designs, Handbook of Combinatorics, pp. 693-745. Elsevier, Amsterdam (1995)

13. Brouwer, A.E., Shearer, J.B., Sloane, N.J.A., Smith, W.D.: A new table of constant weight codes. IEEE Trans. Inform. Theory 36(6), 1334-1380 (1990)

14. Cahill, J., Casazza, P.G., Haas, J.I., Tremain, J.: Constructions of biangular tight frames and their relationships with equiangular tight frames. In: Frames and Harmonic Analysis. Contemp. Math., vol. 706, pp. 1-19. Amer. Math. Soc., Providence (2018)

15. Cameron, P.J., Goethals, J.-M., Seidel, J.J., Shult, E.E.: Line graphs, root systems, and elliptic geometry. J. Algebra 43(1), 305-327 (1976)

16. Cohn, H., Jiao, Y., Kumar, A., Torquato, S.: Rigidity of spherical codes. Geom. Topol. 15(4), 2235-2273 (2011)

17. Conway, J.H., Hardin, R.H., Sloane, N.J.A.: Packing lines, planes, etc.: packings in Grassmannian spaces. Experiment. Math. 5(2), 139-159 (1996)

18. Conway, J.H., Sloane, N.J.A.: Sphere Packings, Lattices and Groups. Grundlehren der Mathematischen Wissenschaften, vol. 290. Springer, New York (1999)

19. Delsarte, P., Goethals, J.M., Seidel, J.J.: Bounds on systems of lines and Jacobi polynomials. Philips Res. Reports 30, 91-105 (1975)

20. Delsarte, P., Goethals, J.M., Seidel, J.J.: Spherical codes and designs. Geometriae Dedicata 6(3), 363388 (1977)

21. Ericson, T., Zinoviev, V.: Codes on Euclidean Spheres. North-Holland Mathematical Library, vol. 63. North-Holland, Amsterdam (2001)

22. Fickus, M., Jasper, J., Mixon, D.G.: Packings in real projective spaces. SIAM J. Appl. Algebra Geom. 2(3), 377-409 (2018)

23. Fickus, M., Mixon, D.G., Tremain, J.C.: Steiner equiangular tight frames. Linear Algebra Appl. 436(5), 1014-1027 (2012)

24. Greaves, G., Koolen, J.H., Munemasa, A., Szöllősi, F.: Equiangular lines in Euclidean spaces. J. Combin. Theory Ser. A 138, 208-235 (2016)

25. Hanaki, A., Miyamoto, I.: Classification of association schemes of small order. Discrete Math. 264(1-3), 75-80 (2003)

26. Hardin, R.H., Sloane, N.J.A.: McLaren's improved snub cube and other new spherical designs in three dimensions. Discrete Comput. Geom. 15(4), 429-441 (1996)

27. Higham, N.J.: Cholesky factorization. WIREs Computational Statistics 1, 251-254 (2009)

28. Holzmann, W.H., Kharaghani, H., Suda, S.: Mutually unbiased biangular vectors and association schemes. In: Algebraic Design Theory and Hadamard Matrices (Lethbridge 2014). Springer Proc. Math. Stat., vol. 133, pp. 149-157. Springer, Cham (2015) 
29. Kantor, W.M.: Codes, quadratic forms and finite geometries. In: Different Aspects of Coding Theory (San Francisco 1995). Proc. Sympos. Appl. Math., vol. 50, pp. 153-177. Amer. Math. Soc., Providence (1995)

30. Kaski, P., Östergård, P.R.J.: Classification Algorithms for Codes and Designs. Algorithms and Computation in Mathematics, vol. 15. Springer, Berlin (2006)

31. Kaski, P., Östergård, P.R.J.: There are exactly five biplanes with $k=11$. J. Combin. Des. 16(2), 117-127 (2008)

32. Lemmens, P.W.H., Seidel, J.J.: Equiangular lines. J. Algebra 24(3), 494-512 (1973)

33. van Lint, J.H., Seidel, J.J.: Equilateral point sets in elliptic geometry. Indag. Math. 28, 335-348 (1966)

34. Lisoněk, P.: New maximal two-distance sets. J. Combin. Theory Ser. A 77(2), 318-338 (1997)

35. McKay, B.D., Piperno, A.: Practical graph isomorphism. II. J. Symbolic Comput. 60, 94-112 (2014)

36. Mixon, D.G., Parshall, H.: The optimal packing of eight points in the real projective plane. Exp. Math. (2019). https://doi.org/10.1080/10586458.2019.1641767

37. Musin, O.R., Nozaki, H.: Bounds on three- and higher-distance sets. European J. Combin. 32(8), 1182-1190 (2011)

38. Neumaier, A.: Lattices of simplex type. SIAM J. Algebraic Discrete Methods 4(2), 145-160 (1983)

39. Nozaki, H.: A generalization of Larman-Rogers-Seidel's theorem. Discrete Math. 311(10-11), 792799 (2011)

40. Nozaki, H., Shinohara, M.: Maximal 2-distance sets containing the regular simplex. Discrete Math. 343(11), \# 112071 (2020)

41. Nozaki, H., Suda, S.: Weighing matrices and spherical codes. J. Algebraic Combin. 42(1), 283-291 (2015)

42. Read, R.C.: Every one a winner or how to avoid isomorphism search when cataloguing combinatorial configurations. Ann. Discrete Math. 2, 107-120 (1978)

43. Shult, E., Yanushka, A.: Near n-gons and line systems. Geom. Dedicata 9(1), 1-72 (1980)

44. Szöllősi, F., Östergård, P.R.J.: Constructions of maximum few-distance sets in Euclidean spaces. Electron. J. Combin. 27(1), \# 1.23 (2020)

45. Waldron, S.F.D.: An Introduction to Finite Tight Frames. Applied and Numerical Harmonic Analysis. Birkhäuser, New York (2018)

Publisher's Note Springer Nature remains neutral with regard to jurisdictional claims in published maps and institutional affiliations. 